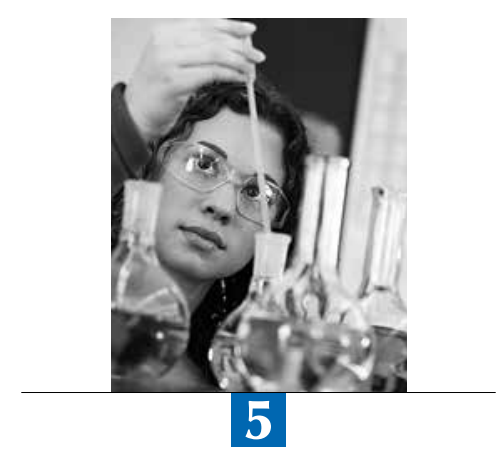

\title{
A Profile \\ of Student Performance in Science
}

This chapter examines student performance in science in PISA 2012. It provides examples of assessment questions, relating them to each PISA proficiency level, discusses gender differences in student performance, compares countries' and economies' performance in science, and highlights trends in science performance up to 2012. 
What can 15-year-old students do in science? This chapter describes how PISA 2012 measures student achievement in science around the world, at the country and regional levels, among boys and girls, and also compares outcomes of PISA 2012 with those of the previous PISA cycles. It provides a few examples of the questions asked in the science assessment.

An understanding of science and technology is central to a young person's preparedness for life in modern society, not least because it empowers individuals to participate in determining public policy where issues of science and technology affect their lives. PISA defines scientific literacy as an individual's scientific knowledge, and use of that knowledge, to identify questions, acquire new knowledge, explain scientific phenomena and draw evidence-based conclusions about science-related issues; understanding of the characteristic features of science as a form of human knowledge and enquiry; awareness of how science and technology shape our material, intellectual and cultural environments; and willingness to engage in science-related issues, and with the ideas of science, as a reflective citizen (OECD, 2007).

Science was the focus of the PISA 2006 survey and a minor domain in PISA 2009 and 2012. Less time was allocated during these latter two assessments than in PISA 2006. Ninety minutes of the assessment time were devoted to science in the last two cycles, allowing for only an update on overall performance rather than the kind of in-depth analysis of knowledge and skills shown in the PISA 2006 report (OECD, 2007).

\section{What the data tell us}

- Nineteen of 64 countries and economies with comparable data show an average annual improvement, 37 show no change, and 8 show a deterioration in their science performance throughout their participation in PISA.

- Hong Kong-China, Ireland, Japan, Korea and Poland performed at or above the OECD average in science in 2006 and by 2012 showed an improvement in science performance of more than two score points per year. Estonia also performed above the OECD average in science in 2006, and between 2009 and 2012 improved its score by 14 points.

- Estonia, Israel, Italy, Poland, Qatar and Singapore reduced the share of students who do not attain the baseline level of proficiency and simultaneously increased the share of top-performing students in science.

- Boys and girls perform similarly in science and, on average, that remained true in 2012. But in Colombia, Japan and Spain, while there was no gender gap in science performance in 2006, a gender gap in favour of boys was observed in 2012.

\section{STUDENT PERFORMANCE IN SCIENCE}

In PISA 2006 the mean science score for OECD countries was initially set at 500 points (for 30 OECD countries), then was re-set at 498 points after taking into account the four newest OECD countries. To help interpret what students' scores mean in substantive terms, the scale is divided into levels of proficiency that indicate the kinds of tasks that students at those levels are capable of completing successfully (OECD, 2006).

\section{Average performance in science}

One way to summarise student performance and to compare the relative standing of countries in science is through countries' mean performance, both relative to each other and to the OECD mean. For PISA 2012, the mean in science for OECD countries increased to 501 points. This establishes the benchmark against which each country and economy's science performance in PISA 2012 is compared.

When interpreting mean performance, only those differences among countries and economies that are statistically significant should be taken into account. Figure I.5.1 shows each country's/economy's mean score and also for which pairs of countries/economies the differences between the means are statistically significant. For each country/economy shown in the middle column, the countries/economies whose mean scores are not statistically significantly different are listed in the right column. In all other cases, country/economy A scores higher than country/economy B if country/ economy $A$ is situated above country/economy $B$ in the middle column, and scores lower if country/economy $A$ is situated below country/economy B. For example: Shanghai-China ranks first on the PISA science scale, but Hong Kong-China, which appears second on the list, cannot be distinguished with confidence from Singapore and Japan, which appear third and fourth, respectively. 
- Figure I.5.1

\section{Comparing countries' and economies' performance in science}

\begin{tabular}{|c|c|c|}
\hline \multirow[b]{3}{*}{$\begin{array}{l}\text { Mean } \\
\text { score }\end{array}$} & \multirow[b]{3}{*}{$\begin{array}{l}\text { Comparison country/ } \\
\text { economy }\end{array}$} & \multirow{2}{*}{$\begin{array}{l}\text { Statistically significantly above the OECD average } \\
\text { Not statistically significantly different from the OECD average } \\
\text { Statistically significantly below the OECD average }\end{array}$} \\
\hline & & \\
\hline & & Countries/economies whose mean score is NOT statistically significantly different from that comparison country's/economy's score \\
\hline 580 & Shanghai-China & \\
\hline 555 & Hong Kong-China & Singapore, Japan \\
\hline 551 & Singapore & Hong Kong-China, Japan \\
\hline 547 & Japan & Hong Kong-China, Singapore, Finland, Estonia, Korea \\
\hline 545 & Finland & Japan, Estonia, Korea \\
\hline 541 & Estonia & Japan, Finland, Korea \\
\hline 538 & Korea & Japan, Finland, Estonia, Viet Nam \\
\hline 528 & Viet Nam & Korea, Poland, Canada, Liechtenstein, Germany, Chinese Taipei, Netherlands, Ireland, Australia, Macao-China \\
\hline 526 & Poland & Viet Nam, Canada, Liechtenstein, Germany, Chinese Taipei, Netherlands, Ireland, Australia, Macao-China \\
\hline 525 & Canada & Viet Nam, Poland, Liechtenstein, Germany, Chinese Taipei, Netherlands, Ireland, Australia \\
\hline 525 & Liechtenstein & Viet Nam, Poland, Canada, Germany, Chinese Taipei, Netherlands, Ireland, Australia, Macao-China \\
\hline 524 & Germany & Viet Nam, Poland, Canada, Liechtenstein, Chinese Taipei, Netherlands, Ireland, Australia, Macao-China \\
\hline 523 & Chinese Taipei & Viet Nam, Poland, Canada, Liechtenstein, Germany, Netherlands, Ireland, Australia, Macao-China \\
\hline 522 & Netherlands & $\begin{array}{l}\text { Viet Nam, Poland, Canada, Liechtenstein, Germany, Chinese Taipei, Ireland, Australia, Macao-China, New Zealand, Switzerland, } \\
\text { United Kingdom }\end{array}$ \\
\hline 522 & Ireland & $\begin{array}{l}\text { Viet Nam, Poland, Canada, Liechtenstein, Germany, Chinese Taipei, Netherlands, Australia, Macao-China, New Zealand, Switzerland, } \\
\text { United Kingdom }\end{array}$ \\
\hline 521 & Australia & Viet Nam, Poland, Canada, Liechtenstein, Germany, Chinese Taipei, Netherlands, Ireland, Macao-China, Switzerland, United Kingdom \\
\hline 521 & Macao-China & Viet Nam, Poland, Liechtenstein, Germany, Chinese Taipei, Netherlands, Ireland, Australia, Switzerland, United Kingdom \\
\hline 516 & New Zealand & Netherlands, Ireland, Switzerland, Slovenia, United Kingdom \\
\hline 515 & Switzerland & Netherlands, Ireland, Australia, Macao-China, New Zealand, Slovenia, United Kingdom, Czech Republic \\
\hline 514 & Slovenia & New Zealand, Switzerland, United Kingdom, Czech Republic \\
\hline 514 & United Kingdom & Netherlands, Ireland, Australia, Macao-China, New Zealand, Switzerland, Slovenia, Czech Republic, Austria \\
\hline 508 & Czech Republic & Switzerland, Slovenia, United Kingdom, Austria, Belgium, Latvia \\
\hline 506 & Austria & United Kingdom, Czech Republic, Belgium, Latvia, France, Denmark, United States \\
\hline 505 & Belgium & Czech Republic, Austria, Latvia, France, United States \\
\hline 502 & Latvia & Czech Republic, Austria, Belgium, France, Denmark, United States, Spain, Lithuania, Norway, Hungary \\
\hline 499 & France & Austria, Belgium, Latvia, Denmark, United States, Spain, Lithuania, Norway, Hungary, Italy, Croatia \\
\hline 498 & Denmark & Austria, Latvia, France, United States, Spain, Lithuania, Norway, Hungary, Italy, Croatia \\
\hline 497 & United States & Austria, Belgium, Latvia, France, Denmark, Spain, Lithuania, Norway, Hungary, Italy, Croatia, Luxembourg, Portugal \\
\hline 496 & Spain & Latvia, France, Denmark, United States, Lithuania, Norway, Hungary, Italy, Croatia, Portugal \\
\hline 496 & Lithuania & Latvia, France, Denmark, United States, Spain, Norway, Hungary, Italy, Croatia, Luxembourg, Portugal \\
\hline 495 & Norway & Latvia, France, Denmark, United States, Spain, Lithuania, Hungary, Italy, Croatia, Luxembourg, Portugal, Russian Federation \\
\hline 494 & Hungary & Latvia, France, Denmark, United States, Spain, Lithuania, Norway, Italy, Croatia, Luxembourg, Portugal, Russian Federation \\
\hline 494 & Italy & France, Denmark, United States, Spain, Lithuania, Norway, Hungary, Croatia, Luxembourg, Portugal \\
\hline 491 & Croatia & France, Denmark, United States, Spain, Lithuania, Norway, Hungary, Italy, Luxembourg, Portugal, Russian Federation, Sweden \\
\hline 491 & Luxembourg & United States, Lithuania, Norway, Hungary, Italy, Croatia, Portugal, Russian Federation \\
\hline 489 & Portugal & United States, Spain, Lithuania, Norway, Hungary, Italy, Croatia, Luxembourg, Russian Federation, Sweden \\
\hline 486 & Russian Federation & Norway, Hungary, Croatia, Luxembourg, Portugal, Sweden \\
\hline 485 & Sweden & Croatia, Portugal, Russian Federation Iceland \\
\hline 478 & Iceland & Sweden, Slovak Republic, Israel \\
\hline 471 & Slovak Republic & Iceland, Israel, Greece, Turkey \\
\hline 470 & Israel & Iceland, Slovak Republic, Greece, Turkey \\
\hline 467 & Greece & Slovak Republic, Israel, Turkey \\
\hline 463 & Turkey & Slovak Republic, Israel, Greece \\
\hline 448 & United Arab Emirates & Bulgaria, Chile, Serbia, Thailand \\
\hline 446 & Bulgaria & United Arab Emirates, Chile, Serbia, Thailand, Romania, Cyprus ${ }^{1,2}$ \\
\hline 445 & Chile & United Arab Emirates, Bulgaria, Serbia, Thailand, Romania \\
\hline 445 & Serbia & United Arab Emirates, Bulgaria, Chile, Thailand, Romania \\
\hline 444 & Thailand & United Arab Emirates, Bulgaria, Chile, Serbia, Romania \\
\hline 439 & Romania & Bulgaria, Chile, Serbia, Thailand, Cyprus 1,2 \\
\hline 438 & Cyprus ${ }^{1,2}$ & Bulgaria, Romania \\
\hline 429 & Costa Rica & Kazakhstan \\
\hline 425 & Kazakhstan & Costa Rica, Malaysia \\
\hline 420 & Malaysia & Kazakhstan, Uruguay, Mexico \\
\hline 416 & Uruguay & Malaysia, Mexico, Montenegro, Jordan \\
\hline 415 & Mexico & Malaysia, Uruguay, Jordan \\
\hline 410 & Montenegro & Uruguay, Jordan, Argentina \\
\hline 409 & Jordan & Uruguay, Mexico, Montenegro, Argentina, Brazil \\
\hline 406 & Argentina & Montenegro, Jordan, Brazil, Colombia, Tunisia, Albania \\
\hline 405 & Brazil & Jordan, Argentina, Colombia, Tunisia \\
\hline 399 & Colombia & Argentina, Brazil, Tunisia, Albania \\
\hline 398 & Tunisia & Argentina, Brazil, Colombia, Albania \\
\hline 397 & Albania & Argentina, Colombia, Tunisia \\
\hline 384 & Qatar & Indonesia \\
\hline 382 & Indonesia & Qatar, Peru \\
\hline 373 & Peru & Indonesia \\
\hline
\end{tabular}

1. Note by Turkey: The information in this document with reference to "Cyprus" relates to the southern part of the Island. There is no single authority representing both Turkish and Greek Cypriot people on the Island. Turkey recognises the Turkish Republic of Northern Cyprus (TRNC). Until a lasting and equitable solution is found within the context of the United Nations, Turkey shall preserve its position concerning the "Cyprus issue".

2. Note by all the European Union Member States of the OECD and the European Union: The Republic of Cyprus is recognised by all members of the United Nations with the exception of Turkey. The information in this document relates to the area under the effective control of the Government of the Republic of Cyprus.

Source: OECD, PISA 2012 Database.

StatLink 解出 http://dx.doi.org/10.1787/888932935629 
Moreover, countries and economies are divided into three broad groups: those whose mean scores are statistically around the OECD mean (highlighted in dark blue), those whose mean scores are above the OECD mean (highlighted in pale blue), and those whose mean scores are below the OECD mean (highlighted in medium blue).

As shown in Figure 1.5.1, five countries and economies outperform all other countries and economies in science in PISA 2012 by about half a standard deviation above the average or more: Shanghai-China (580 points), Hong Kong-China (555 points), Singapore (551 points), Japan (547 points) and Finland (545 points). Shanghai-China has a mean score of 580, which is more than three-quarters of a proficiency level above the average of 501 score points in PISA 2012. Other countries with mean performances above the average include Estonia, Korea, Viet Nam, Poland, Canada, Liechtenstein, Germany, Chinese Taipei, the Netherlands, Ireland, Australia, Macao-China, New Zealand, Switzerland, Slovenia, the United Kingdom, the Czech Republic and Belgium. Countries that performed around the average include Austria, Latvia, France, Denmark and the United States. Thirty-seven participating countries and economies have a mean score that is below the OECD average.

The gap in performance between the highest- and the lowest-performing OECD countries is 132 score points. That is, while the average score of the highest-performing OECD country, Japan (547), is slightly more than half a standard deviation above the OECD average, the average score of the lowest-performing OECD country, Mexico (415 points) is more than three-quarters of one standard deviation below the OECD average. But the performance difference observed among partner countries and economies is even larger, with a 207 score-point difference between Shanghai-China (580 points) and Peru (373 points).

Because the figures are derived from samples, it is not possible to determine a country's/economy's precise ranking among all participating countries and economies. However, it is possible to determine with confidence a range of rankings in which the country's/economy's performance level lies (Figure 1.5.2). For entities other than those for which full samples were drawn (i.e. Shanghai-China, Hong Kong-China, Chinese Taipei and Macao-China) is not possible to calculate a rank order but the mean score provides a possibility to position subnational entities against the performance of the countries and economies. For example Western Australia shows a score just below the performance of top-performer Korea.

\section{Trends in average science performance}

The change in a school system's average performance over time indicates how and to what extent the system is progressing towards achieving the goal of providing all students with the knowledge and skills needed to become full participants in a knowledge-based society. PISA 2012 science results can be compared with those from PISA 2009 and PISA 2006, when science was first a major domain. PISA 2012 results for 54 countries and economies can be compared with data from both PISA 2009 and PISA 2006; trends for nine countries and economies can be observed using data from PISA 2009 and PISA 2012; and trends for one country can be observed using data from PISA 2006 and PISA 2012. The following trends in average performance are presented as the annualised change for these 64 countries and economies the average yearly change in science performance observed in a country or economy throughout its participation in PISA. (For further details on the estimation of the annualised change, see Annex A5). ${ }^{1}$

On average across OECD countries, science performance has remained broadly stable since 2006. Among the 64 countries and economies with annualised change, 19 countries and economies saw improvements in their science performance. Figure I.5.3 shows that the annualised change was largest in Kazakhstan (at an annual increase of eight score points per year), Turkey (six score points per year), Qatar and Poland (five and four points per year, respectively), Thailand, Romania, Singapore and Italy (three points per year). For example, the average 15-year-old student in Turkey scored 424 points in the PISA 2006 science assessment; three years later, the average student scored 454 points and, in 2012, he or she scored 463 points. Similarly, in Poland in 2006, the average student scored at the OECD average of 498 points in science, improved to 508 points in 2009, then improved again to score 526 points in 2012 (Table I.5.3b).

Improvements of more than two score points per year were observed in Israel, Korea, Japan, Dubai (United Arab Emirates), Portugal, Brazil, Ireland, Tunisia, Hong Kong-China and Latvia. Annualised improvement in science was also seen in Macao-China.

The average change observed over successive PISA cycles does not capture the extent to which this change is steady, or whether it is decelerating or accelerating. The rate of acceleration of improvement may be steady, in which case the science skills of a country's/economy's students improved at a steady pace between 2006 and 2012. The rate may also be accelerating, in which case the improvement between 2009 and 2012 is greater than that between 2006 and 2009; or the rate could be decelerating, in which case there was less of an improvement observed between 2009 and 2012 than between 2006 and 2009. 
- Figure 1.5.2 [Part 1/3] Ш

\section{Science performance among PISA 2012 participants, at national and regional levels}

\begin{tabular}{|c|c|c|c|c|c|}
\hline & \multicolumn{5}{|c|}{ Science scale } \\
\hline & \multirow[b]{3}{*}{ Mean score } & \multicolumn{4}{|c|}{ Range of ranks } \\
\hline & & \multicolumn{2}{|c|}{ OECD countries } & \multicolumn{2}{|c|}{ All countries/economies } \\
\hline & & Upper rank & Lower rank & Upper rank & Lower rank \\
\hline Shanghai-China & 580 & & & 1 & 1 \\
\hline Hong Kong-China & 555 & & & 2 & 3 \\
\hline Singapore & 551 & & & 2 & 4 \\
\hline Japan & 547 & 1 & 3 & 3 & 6 \\
\hline Finland & 545 & 1 & 3 & 4 & 6 \\
\hline Estonia & 541 & 2 & 4 & 5 & 7 \\
\hline Korea & 538 & 2 & 4 & 5 & 8 \\
\hline Western Australia (Australia) & 535 & & & & \\
\hline Australian Capital Territory (Australia) & 534 & & & & \\
\hline Trento (Italy) & 533 & & & & \\
\hline Friuli Venezia Giulia (Italy) & 531 & & & & \\
\hline Veneto (Italy) & 531 & & & & \\
\hline Lombardia (Italy) & 529 & & & & \\
\hline Viet Nam & 528 & & & 7 & 15 \\
\hline Massachusetts (United States) & 527 & & & & \\
\hline Poland & 526 & 5 & 9 & 8 & 16 \\
\hline New South Wales (Australia) & 526 & & & & \\
\hline Canada & 525 & 5 & 8 & 8 & 14 \\
\hline Liechtenstein & 525 & & & 8 & 17 \\
\hline Germany & 524 & 5 & 10 & 8 & 17 \\
\hline Chinese Taipei & 523 & & & 9 & 17 \\
\hline Netherlands & 522 & 5 & 11 & 8 & 18 \\
\hline Ireland & 522 & 6 & 11 & 10 & 18 \\
\hline Australia & 521 & 7 & 11 & 11 & 18 \\
\hline Connecticut (United States) & 521 & & & & \\
\hline Macao-China & 521 & & & 13 & 17 \\
\hline Flemish community (Belgium) & 519 & & & & \\
\hline Castile and Leon (Spain) & 519 & & & & \\
\hline Bolzano (Italy) & 519 & & & & \\
\hline Queensland (Australia) & 519 & & & & \\
\hline Victoria (Australia) & 518 & & & & \\
\hline Madrid (Spain) & 517 & & & & \\
\hline Asturias (Spain) & 517 & & & & \\
\hline England (United Kingdom) & 516 & & & & \\
\hline New Zealand & 516 & 10 & 14 & 17 & 21 \\
\hline Switzerland & 515 & 10 & 15 & 17 & 22 \\
\hline Slovenia & 514 & 11 & 14 & 18 & 21 \\
\hline Navarre (Spain) & 514 & & & & \\
\hline United Kingdom & 514 & 10 & 15 & 16 & 22 \\
\hline Scotland (United Kingdom) & 513 & & & & \\
\hline South Australia (Australia) & 513 & & & & \\
\hline Emilia Romagna (Italy) & 512 & & & & \\
\hline Galicia (Spain) & 512 & & & & \\
\hline La Rioja (Spain) & 510 & & & & \\
\hline Piemonte (Italy) & 509 & & & & \\
\hline Czech Republic & 508 & 14 & 17 & 21 & 25 \\
\hline Valle d'Aosta (Italy) & 508 & & & & \\
\hline German-speaking community (Belgium) & 508 & & & & \\
\hline Northern Ireland (United Kingdom) & 507 & & & & \\
\hline Marche (Italy) & 507 & & & & \\
\hline Austria & 506 & 15 & 18 & 22 & 26 \\
\hline Basque Country (Spain) & 506 & & & & \\
\hline Belgium & 505 & 15 & 18 & 22 & 25 \\
\hline Aragon (Spain) & 504 & & & & \\
\hline Latvia & 502 & & & 23 & 29 \\
\hline Umbria (Italy) & 501 & & & & \\
\hline Liguria (Italy) & 501 & & & & \\
\hline Toscana (Italy) & 501 & & & & \\
\hline Cantabria (Spain) & 501 & & & & \\
\hline Tasmania (Australia) & 500 & & & & \\
\hline France & 499 & 17 & 22 & 24 & 31 \\
\hline Denmark & 498 & 17 & 23 & 24 & 32 \\
\hline United States & 497 & 17 & 25 & 24 & 35 \\
\hline
\end{tabular}

Notes: OECD countries are shown in bold black. Partner countries are shown in bold blue. Participating economies and subnational entities that are not included in national results are shown in bold blue italics. Regions are shown in black italics (OECD countries) or blue italics (partner countries).

1. Note by Turkey: The information in this document with reference to "Cyprus" relates to the southern part of the Island. There is no single authority representing both Turkish and Greek Cypriot people on the Island. Turkey recognises the Turkish Republic of Northern Cyprus (TRNC). Until a lasting and equitable solution is found within the context of the United Nations, Turkey shall preserve its position concerning the "Cyprus issue".

2. Note by all the European Union Member States of the OECD and the European Union: The Republic of Cyprus is recognised by all members of the United Nations with the exception of Turkey. The information in this document relates to the area under the effective control of the Government of the Republic of Cyprus.

Countries, economies and subnational entities are ranked in descending order of mean science performance.

Source: OECD, PISA 2012 Database.

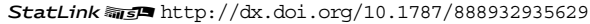


- Figure I.5.2 [Part 2/3]

\section{Science performance among PISA 2012 participants, at national and regional levels}

\begin{tabular}{|c|c|c|c|c|c|}
\hline & \multicolumn{5}{|c|}{ Science scale } \\
\hline & \multirow[b]{3}{*}{ Mean score } & \multicolumn{4}{|c|}{ Range of ranks } \\
\hline & & \multicolumn{2}{|c|}{ OECD countries } & \multicolumn{2}{|c|}{ All countries/economies } \\
\hline & & Upper rank & Lower rank & Upper rank & Lower rank \\
\hline Spain & 496 & 18 & 23 & 26 & 33 \\
\hline Lithuania & 496 & & & 26 & 34 \\
\hline Norway & 495 & 19 & 26 & 26 & 36 \\
\hline Hungary & 494 & 19 & 26 & 27 & 36 \\
\hline Alentejo (Portugal) & 494 & & & & \\
\hline Italy & 494 & 20 & 26 & 28 & 35 \\
\hline Catalonia (Spain) & 492 & & & & \\
\hline Croatia & 491 & & & 29 & 38 \\
\hline Luxembourg & 491 & 23 & 26 & 32 & 36 \\
\hline Wales (United Kingdom) & 491 & & & & \\
\hline Portugal & 489 & 22 & 27 & 30 & 38 \\
\hline French community (Belgium) & 487 & & & & \\
\hline Russian Federation & 486 & & & 34 & 38 \\
\hline Andalusia (Spain) & 486 & & & & \\
\hline Florida (United States) & 485 & & & & \\
\hline Sweden & 485 & 26 & 28 & 36 & 39 \\
\hline Lazio (Italy) & 484 & & & & \\
\hline Puglia (Italy) & 483 & & & & \\
\hline Northern Territory (Australia) & 483 & & & & \\
\hline Balearic Islands (Spain) & 483 & & & & \\
\hline Extremadura (Spain) & 483 & & & & \\
\hline Abruzzo (Italy) & 482 & & & & \\
\hline Perm Territory region (Russian Federation) & 480 & & & & \\
\hline Murcia (Spain) & 479 & & & & \\
\hline Iceland & 478 & 28 & 29 & 38 & 40 \\
\hline Dubai (United Arab Emirates) & 474 & & & & \\
\hline Sardegna (Italy) & 473 & & & & \\
\hline Slovak Republic & 471 & 28 & 31 & 39 & 42 \\
\hline Israel & 470 & 28 & 32 & 39 & 43 \\
\hline Molise (Italy) & 468 & & & & \\
\hline Greece & 467 & 29 & 32 & 40 & 43 \\
\hline Basilicata (Italy) & 465 & & & & \\
\hline Turkey & 463 & 30 & 32 & 41 & 43 \\
\hline Campania (Italy) & 457 & & & & \\
\hline Sicilia (Italy) & 454 & & & & \\
\hline Sharjah (United Arab Emirates) & 450 & & & & \\
\hline United Arab Emirates & 448 & & & 44 & 47 \\
\hline Bulgaria & 446 & & & 44 & 49 \\
\hline Chile & 445 & 33 & 33 & 44 & 48 \\
\hline Serbia & 445 & & & 44 & 49 \\
\hline Thailand & 444 & & & 44 & 49 \\
\hline Abu Dhabi (United Arab Emirates) & 440 & & & & \\
\hline Romania & 439 & & & 47 & 50 \\
\hline Cyprus $1,2^{1,2}$ & 438 & & & 48 & 50 \\
\hline Jalisco (Mexico) & 436 & & & & \\
\hline Nuevo León (Mexico) & 435 & & & & \\
\hline Aguascalientes (Mexico) & 435 & & & & \\
\hline Querétaro (Mexico) & 432 & & & & \\
\hline Ras Al Khaimah (United Arab Emirates) & 431 & & & & \\
\hline Calabria (Italy) & 431 & & & & \\
\hline Colima (Mexico) & 429 & & & & \\
\hline Costa Rica & 429 & & & 51 & 52 \\
\hline Chihuahua (Mexico) & 429 & & & & \\
\hline Manizales (Colombia) & 429 & & & & \\
\hline Espírito Santo (Brazil) & 428 & & & & \\
\hline Distrito Federal (Mexico) & 427 & & & & \\
\hline Fujairah (United Arab Emirates) & 425 & & & & \\
\hline Morelos (Mexico) & 425 & & & & \\
\hline Kazakhstan & 425 & & & 51 & 53 \\
\hline Ciudad Autónoma de Buenos Aires (Argentina) & 425 & & & & \\
\hline Puebla (Mexico) & 423 & & & & \\
\hline Durango (Mexico) & 423 & & & & \\
\hline Federal District (Brazil) & 423 & & & & \\
\hline
\end{tabular}

Notes: OECD countries are shown in bold black. Partner countries are shown in bold blue. Participating economies and subnational entities that are not included in national results are shown in bold blue italics. Regions are shown in black italics (OECD countries) or blue italics (partner countries).

1. Note by Turkey: The information in this document with reference to "Cyprus" relates to the southern part of the Island. There is no single authority representing both Turkish and Greek Cypriot people on the Island. Turkey recognises the Turkish Republic of Northern Cyprus (TRNC). Until a lasting and equitable solution is found within the context of the United Nations, Turkey shall preserve its position concerning the "Cyprus issue".

2. Note by all the European Union Member States of the OECD and the European Union: The Republic of Cyprus is recognised by all members of the United Nations with the exception of Turkey. The information in this document relates to the area under the effective control of the Government of the Republic of Cyprus.

Countries, economies and subnational entities are ranked in descending order of mean science performance.

Source: OECD, PISA 2012 Database.

StatLink 前占 $\mathrm{http}: / / \mathrm{dx}$.doi.org/10.1787/888932935629 
- Figure I.5.2 [Part 3/3]

\section{Science performance among PISA 2012 participants, at national and regional levels}

\begin{tabular}{|c|c|c|c|c|c|}
\hline & \multicolumn{5}{|c|}{ Science scale } \\
\hline & \multirow[b]{3}{*}{ Mean score } & \multicolumn{4}{|c|}{ Range of ranks } \\
\hline & & \multicolumn{2}{|c|}{ OECD countries } & \multicolumn{2}{|c|}{ All countries/economies } \\
\hline & & Upper rank & Lower rank & Upper rank & Lower rank \\
\hline Coahuila (Mexico) & 421 & & & & \\
\hline Mexico (Mexico) & 421 & & & & \\
\hline Ajman (United Arab Emirates) & 420 & & & & \\
\hline Minas Gerais (Brazil) & 420 & & & & \\
\hline Malaysia & 420 & & & 52 & 55 \\
\hline Rio Grande do Sul (Brazil) & 419 & & & & \\
\hline Baja California Sur (Mexico) & 418 & & & & \\
\hline Santa Catarina (Brazil) & 418 & & & & \\
\hline Medellin (Colombia) & 418 & & & & \\
\hline Baja California (Mexico) & 417 & & & & \\
\hline São Paulo (Brazil) & 417 & & & & \\
\hline Quintana Roo (Mexico) & 416 & & & & \\
\hline San Luis Potosí (Mexico) & 416 & & & & \\
\hline Uruguay & 416 & & & 53 & 56 \\
\hline Paraná (Brazil) & 416 & & & & \\
\hline Umm Al Quwain (United Arab Emirates) & 415 & & & & \\
\hline Yucatán (Mexico) & 415 & & & & \\
\hline Mexico & 415 & 34 & 34 & 54 & 56 \\
\hline Mato Grosso do Sul (Brazil) & 415 & & & & \\
\hline Tamaulipas (Mexico) & 414 & & & & \\
\hline Tlaxcala (Mexico) & 412 & & & & \\
\hline Paraiba (Brazil) & 412 & & & & \\
\hline Bogota (Colombia) & 411 & & & & \\
\hline Hidalgo (Mexico) & 411 & & & & \\
\hline Montenegro & 410 & & & 56 & 58 \\
\hline Jordan & 409 & & & 55 & 59 \\
\hline Sinaloa (Mexico) & 408 & & & & \\
\hline Nayarit (Mexico) & 407 & & & & \\
\hline Argentina & 406 & & & 56 & 61 \\
\hline Campeche (Mexico) & 405 & & & & \\
\hline Brazil & 405 & & & 57 & 60 \\
\hline Guanajuato (Mexico) & 404 & & & & \\
\hline Piauí (Colombia) & 403 & & & & \\
\hline Zacatecas (Mexico) & 402 & & & & \\
\hline Cali (Brazil) & 402 & & & & \\
\hline Veracruz (Mexico) & 401 & & & & \\
\hline Rio de Janeiro (Brazil) & 401 & & & & \\
\hline Colombia & 399 & & & 59 & 62 \\
\hline Tunisia & 398 & & & 59 & 62 \\
\hline Albania & 397 & & & 60 & 62 \\
\hline Goiás (Brazil) & 396 & & & & \\
\hline Sergipe (Brazil) & 394 & & & & \\
\hline Tabasco (Mexico) & 391 & & & & \\
\hline Bahia (Brazil) & 390 & & & & \\
\hline Rondônia (Brazil) & 389 & & & & \\
\hline Rio Grande do Norte (Brazil) & 387 & & & & \\
\hline Ceará (Brazil) & 386 & & & & \\
\hline Qatar & 384 & & & 63 & 64 \\
\hline Amapá (Brazil) & 382 & & & & \\
\hline Indonesia & 382 & & & 63 & 64 \\
\hline Mato Grosso (Brazil) & 381 & & & & \\
\hline Acre (Brazil) & 380 & & & & \\
\hline Tocantins (Brazil) & 378 & & & & \\
\hline Chiapas (Mexico) & 377 & & & & \\
\hline Pará (Brazil) & 377 & & & & \\
\hline Amazonas (Brazil) & 376 & & & & \\
\hline Roraima (Brazil) & 375 & & & & \\
\hline Pernambuco (Brazil) & 374 & & & & \\
\hline Peru & 373 & & & 65 & 65 \\
\hline Guerrero (Mexico) & 372 & & & & \\
\hline Maranhão (Brazil) & 359 & & & & \\
\hline Alagoas (Brazil) & 346 & & & & \\
\hline
\end{tabular}

Notes: OECD countries are shown in bold black. Partner countries are shown in bold blue. Participating economies and subnational entities that are not included in national results are shown in bold blue italics. Regions are shown in black italics (OECD countries) or blue italics (partner countries).

1. Note by Turkey: The information in this document with reference to "Cyprus" relates to the southern part of the Island. There is no single authority representing both Turkish and Greek Cypriot people on the Island. Turkey recognises the Turkish Republic of Northern Cyprus (TRNC). Until a lasting and equitable solution is found within the context of the United Nations, Turkey shall preserve its position concerning the "Cyprus issue".

2. Note by all the European Union Member States of the OECD and the European Union: The Republic of Cyprus is recognised by all members of the United Nations with the exception of Turkey. The information in this document relates to the area under the effective control of the Government of the Republic of Cyprus.

Countries, economies and subnational entities are ranked in descending order of mean science performance.

Source: OECD, PISA 2012 Database.

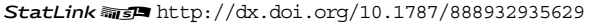




\section{- Figure I.5.3 -}

\section{Annualised change in science performance throughout participation in PISA}

Science score-point difference associated with one calendar year

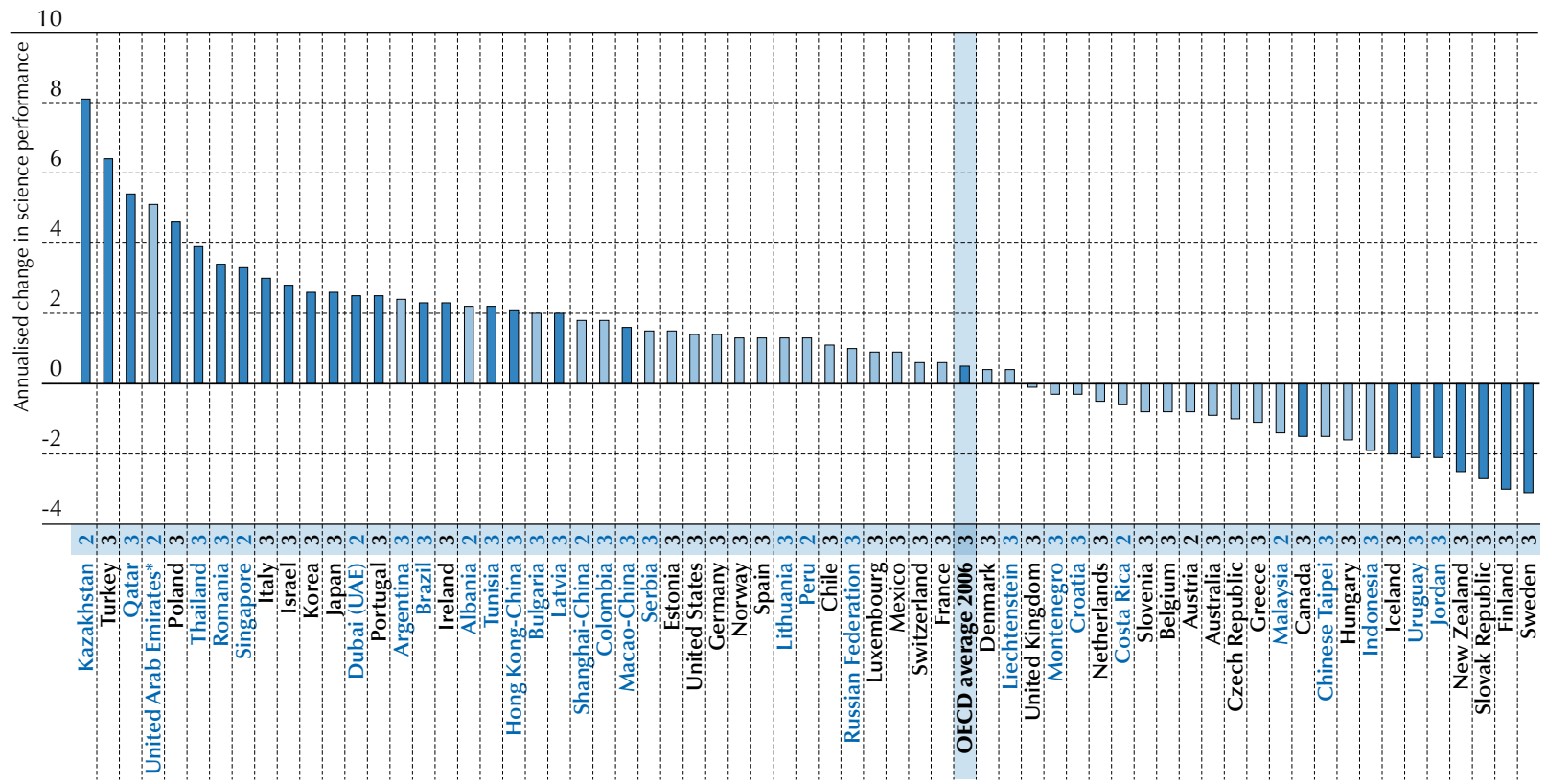

* United Arab Emirates excluding Dubai.

Notes: Statistically significant score point changes are marked in a darker tone (see Annex A3).

The number of comparable science scores used to calculate the annualised change is shown in next to the country/economy name.

The annualised change is the average annual change in PISA score points from a country's/economy's earliest participation in PISA to PISA 2012. It is calculated taking into account all of a country's/economy's participation in PISA. For more details on the calculation of the annualised change, see Annex A5. OECD average 2006 compares only OECD countries with comparable science scores since 2006.

Countries and economies are ranked in descending order of the annualised change in science performance.

Source: OECD, PISA 2012 Database, Table I.5.3b.

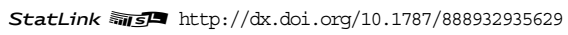

Results on the rate of acceleration of a country's/economy's improvement can be calculated only for the 54 countries and economies that participated in PISA 2006, PISA 2009 and PISA 2012, 16 of which saw an annualised improvement in science performance during the period. Of these 16 countries, Macao-China shows greater improvement between 2009 and 2012 than between 2006 and 2009. Improvements in science performance decelerated in Brazil, Portugal, Qatar, Tunisia and Turkey, where the observed improvement between 2009 and 2012 was smaller than that observed between 2006 and 2009. For the remaining countries, the annualised improvement is relatively similar between the 2006-09 and 2009-12 periods. Other countries and economies show no overall average annual improvement in performance, but do show notable improvements in science performance between PISA 2009 and PISA 2012. Such is the case of Estonia, where science performance improved by 14 score points as well as Luxembourg and Montenegro (Figure I.5.4).

At any point in time, countries and economies share similar levels of performance in science with other countries and economies. But as time passes and school systems evolve, some countries and economies may improve their performance while others may not. Figure I.5.5 shows, for each country and economy with comparable results in 2006 and 2012, those other countries and economies that had similar performance in 2006 but whose performance improved or deteriorated by 2012. For example, in 2006, Japan was similar in science performance to New Zealand, Chinese Taipei, Australia, Canada, the Netherlands, Liechtenstein, Hong Kong-China, Estonia and Korea; but after its annualised improvement of 2.6 score points per year, it scored higher in science than New Zealand, Chinese Taipei, Australia, Canada, the Netherlands and Liechtenstein in 2012. In 2006, Germany had lower scores in science than New Zealand, Chinese Taipei and Canada; but by 2012, its performance was similar to those countries' performance. Along the same lines, Romania had similar levels of performance as Uruguay, Jordan, Montenegro, Mexico, Thailand and Bulgaria in 2006. By 2012, Romania showed better performance than Uruguay, Jordan, Montenegro and Mexico, and had attained similar levels of performance as Chile and Serbia, both of which had higher scores in science than Romania did in 2006. Estonia's improvement in PISA and recent educational policies and programmes is outlined in Box I.5.1. 
- Figure I.5.4 -

\section{Curvilinear trajectories of average science performance across PISA assessments}

Rate of acceleration or deceleration in performance (quadratic term)

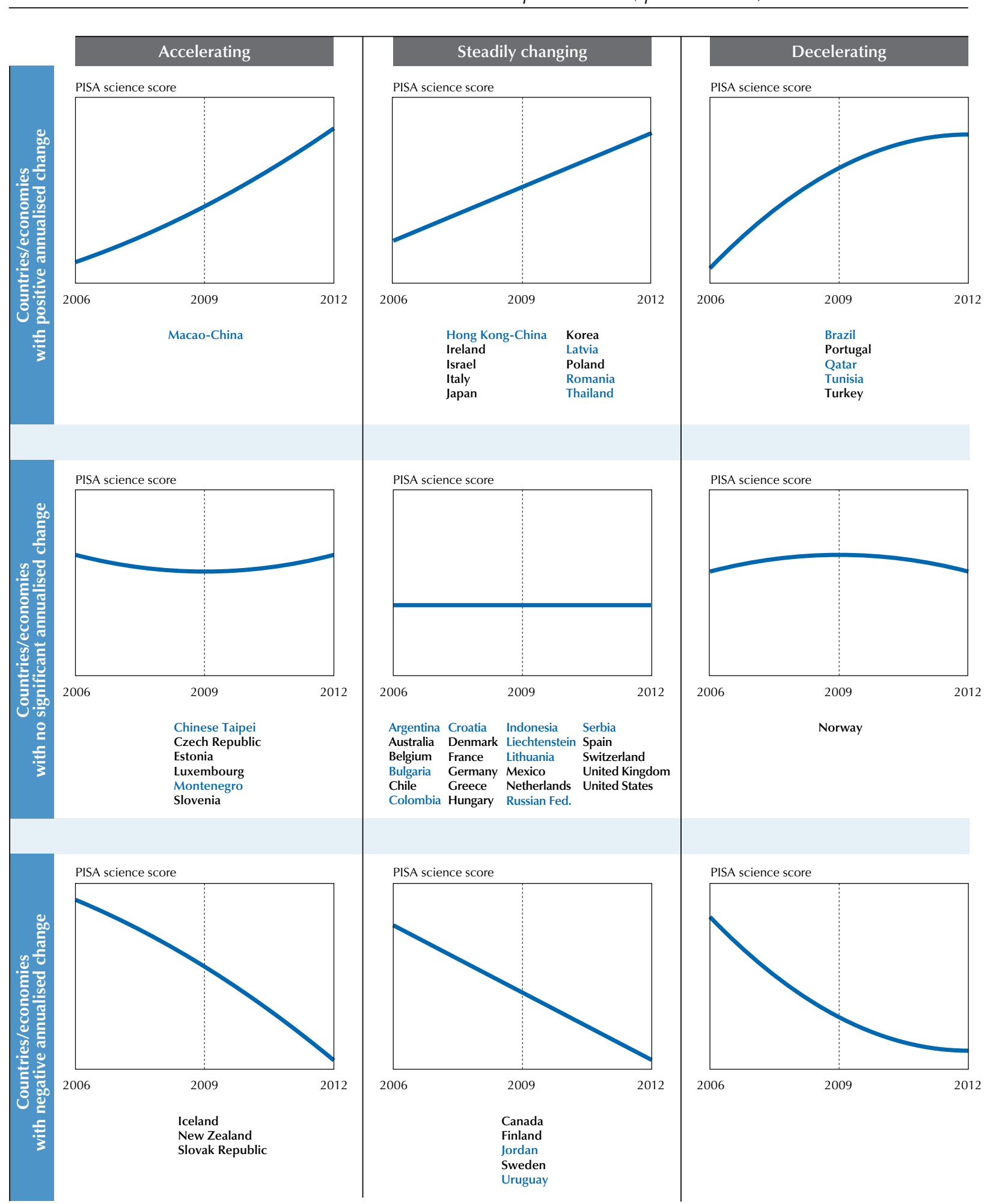

Notes: Figures are for illustrative purposes only. Countries and economies are grouped according to the direction and significance of their annualised change and their rate of acceleration.

Countries and economies with data from only one PISA assessments other than 2012 are excluded.

Source: OECD, PISA 2012 Database, Table I.5.3b.

StatLink त्ता st http://dx.doi.org/10.1787/888932935629 
- Figure I.5.5 [Part 1/4]

Multiple comparisons of science performance between 2006 and 2012

\begin{tabular}{|c|c|c|c|c|c|}
\hline & $\begin{array}{l}\text { Science } \\
\text { performance } \\
\text { in } 2006\end{array}$ & $\begin{array}{c}\begin{array}{c}\text { Science } \\
\text { performance } \\
\text { in } 2012\end{array} \\
\end{array}$ & $\begin{array}{c}\text { Countries/economies with similar } \\
\text { performance in } 2006 \\
\text { but lower performance in } 2012\end{array}$ & $\begin{array}{c}\text { Countries/economies with similar } \\
\text { performance in } 2006 \\
\text { and similar performance in } 2012\end{array}$ & $\begin{array}{c}\text { Countries/economies with similar } \\
\text { performance in } 2006 \\
\text { but higher performance in } 2012\end{array}$ \\
\hline Hong Kong-China & 542 & 555 & Chinese Taipei, Canada & Japan & \\
\hline Japan & 531 & 547 & $\begin{array}{l}\text { New Zealand, Chinese Taipei, Australia, } \\
\text { Canada, Netherlands, Liechtenstein }\end{array}$ & Hong Kong-China, Estonia, Korea & \\
\hline Finland & 563 & 545 & & & \\
\hline Estonia & 531 & 541 & $\begin{array}{l}\text { New Zealand, Chinese Taipei, Australia, } \\
\text { Canada, Netherlands, Liechtenstein }\end{array}$ & Japan, Korea & \\
\hline Korea & 522 & 538 & $\begin{array}{l}\text { New Zealand, United Kingdom, } \\
\text { Germany, Austria, Czech Republic, } \\
\text { Chinese Taipei, Australia, Netherlands, } \\
\text { Switzerland, Slovenia }\end{array}$ & Estonia, Japan, Liechtenstein & \\
\hline Poland & 498 & 526 & $\begin{array}{l}\text { United States, Croatia, Latvia, } \\
\text { Slovak Republic, Lithuania, France, } \\
\text { Sweden, Hungary, Spain, Denmark, } \\
\text { Iceland }\end{array}$ & Ireland & \\
\hline Canada & 534 & 525 & New Zealand & Chinese Taipei, Australia & Hong Kong-China, Estonia, Japan \\
\hline Liechtenstein & 522 & 525 & $\begin{array}{l}\text { Austria, Czech Republic, Belgium, } \\
\text { Slovenia }\end{array}$ & $\begin{array}{l}\text { New Zealand, United Kingdom, } \\
\text { Germany, Chinese Taipei, Australia, } \\
\text { Ireland, Netherlands, Switzerland, Korea }\end{array}$ & Estonia, Japan \\
\hline Germany & 516 & 524 & $\begin{array}{l}\text { Austria, Czech Republic, Hungary, } \\
\text { Belgium, Slovenia }\end{array}$ & $\begin{array}{l}\text { United Kingdom, Australia, Ireland, } \\
\text { Macao-China, Netherlands, Switzerland, } \\
\text { Liechtenstein }\end{array}$ & Korea \\
\hline Chinese Taipei & 532 & 523 & & $\begin{array}{l}\text { New Zealand, Australia, Canada, } \\
\text { Netherlands, Liechtenstein }\end{array}$ & Hong Kong-China, Estonia, Japan, Korea \\
\hline Netherlands & 525 & 522 & Czech Republic & $\begin{array}{l}\text { New Zealand, Germany, Chinese Taipei, } \\
\text { Australia, Slovenia, Liechtenstein }\end{array}$ & Estonia, Japan, Korea \\
\hline Ireland & 508 & 522 & $\begin{array}{l}\text { Austria, Czech Republic, Sweden, } \\
\text { Hungary, Belgium }\end{array}$ & $\begin{array}{l}\text { Poland, United Kingdom, Germany, } \\
\text { Macao-China, Switzerland, Liechtenstein }\end{array}$ & \\
\hline Australia & 527 & 521 & & $\begin{array}{l}\text { New Zealand, Germany, Chinese Taipei, } \\
\text { Canada, Netherlands, Liechtenstein }\end{array}$ & Estonia, Japan, Korea \\
\hline Macao-China & 511 & 521 & $\begin{array}{l}\text { Austria, Czech Republic, Hungary, } \\
\text { Belgium }\end{array}$ & $\begin{array}{l}\text { United Kingdom, Germany, Ireland, } \\
\text { Switzerland }\end{array}$ & \\
\hline New Zealand & 530 & 516 & & $\begin{array}{l}\text { Chinese Taipei, Australia, Netherlands, } \\
\text { Liechtenstein }\end{array}$ & Estonia, Japan, Canada, Korea \\
\hline Switzerland & 512 & 515 & Sweden, Hungary, Belgium & $\begin{array}{l}\text { United Kingdom, Germany, Austria, } \\
\text { Czech Republic, Ireland, Macao-China, } \\
\text { Slovenia, Liechtenstein }\end{array}$ & Korea \\
\hline Slovenia & 519 & 514 & Austria & $\begin{array}{l}\text { United Kingdom, Czech Republic, } \\
\text { Netherlands, Switzerland }\end{array}$ & Germany, Liechtenstein, Korea \\
\hline United Kingdom & 515 & 514 & & $\begin{array}{l}\text { Germany, Austria, Czech Republic, } \\
\text { Ireland, Macao-China, Belgium, } \\
\text { Switzerland, Slovenia, Liechtenstein }\end{array}$ & Korea \\
\hline Czech Republic & 513 & 508 & Sweden, Hungary & $\begin{array}{l}\text { United Kingdom, Austria, Belgium, } \\
\text { Switzerland, Slovenia }\end{array}$ & $\begin{array}{l}\text { Germany, Ireland, Macao-China, } \\
\text { Netherlands, Liechtenstein, Korea }\end{array}$ \\
\hline Austria & 511 & 506 & Sweden, Hungary & $\begin{array}{l}\text { United Kingdom, Czech Republic, } \\
\text { Belgium, Switzerland }\end{array}$ & $\begin{array}{l}\text { Germany, Ireland, Macao-China, } \\
\text { Slovenia, Liechtenstein, Korea }\end{array}$ \\
\hline Belgium & 510 & 505 & Sweden, Hungary & $\begin{array}{l}\text { United Kingdom, Austria, } \\
\text { Czech Republic }\end{array}$ & $\begin{array}{l}\text { Germany, Ireland, Macao-China, } \\
\text { Switzerland, Liechtenstein }\end{array}$ \\
\hline Latvia & 490 & 502 & $\begin{array}{l}\text { Slovak Republic, Luxembourg, Iceland, } \\
\text { Russian Federation }\end{array}$ & $\begin{array}{l}\text { United States, Croatia, Lithuania, France, } \\
\text { Spain, Denmark, Norway }\end{array}$ & Poland \\
\hline France & 495 & 499 & Slovak Republic, Sweden, Iceland & $\begin{array}{l}\text { United States, Croatia, Latvia, Lithuania, } \\
\text { Hungary, Spain, Denmark, Norway }\end{array}$ & Poland \\
\hline Denmark & 496 & 498 & Slovak Republic, Sweden, Iceland & $\begin{array}{l}\text { United States, Croatia, Latvia, Lithuania, } \\
\text { France, Hungary, Spain, Norway }\end{array}$ & Poland \\
\hline United States & 489 & 497 & Slovak Republic, Iceland & $\begin{array}{l}\text { Croatia, Latvia, Luxembourg, Lithuania, } \\
\text { France, Spain, Denmark, Norway, } \\
\text { Russian Federation }\end{array}$ & Poland \\
\hline
\end{tabular}

Note: Only countries and economies that participated in the PISA 2006 and PISA 2012 assessments are shown.

Countries and economies are ranked in descending order of their mean science performance in PISA 2012.

Source: OECD, PISA 2012 Database, Table 1.5.3b

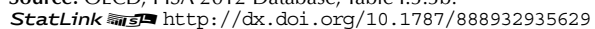


- Figure I.5.5 [Part 2/4]

Multiple comparisons of science performance between 2006 and 2012

\begin{tabular}{|c|c|c|c|c|c|c|}
\hline $\begin{array}{c}\text { Countries/economies with } \\
\text { lower performance in } 2006 \\
\text { but similar performance } \\
\text { in } 2012\end{array}$ & $\begin{array}{l}\text { Countries/economies with } \\
\text { lower performance in } 2006 \\
\text { but higher performance } \\
\text { in } 2012\end{array}$ & $\begin{array}{c}\text { Countries/economies with } \\
\text { higher performance in } 2006 \\
\text { but with similar performance } \\
\text { in } 2012\end{array}$ & $\begin{array}{l}\text { Countries/economies with } \\
\text { higher performance in } 2006 \\
\text { but lower performance } \\
\text { in } 2012\end{array}$ & $\begin{array}{c}\text { Science } \\
\text { performance } \\
\text { in } 2012\end{array}$ & $\begin{array}{c}\text { Science } \\
\text { performance } \\
\text { in } 2006\end{array}$ & \\
\hline & & & Finland & 555 & 542 & Hong Kong-China \\
\hline & & Finland & & 547 & 531 & Japan \\
\hline \multirow[t]{2}{*}{ Estonia, Japan, Korea } & Hong Kong-China & & & 545 & 563 & Finland \\
\hline & & Finland & & 541 & 531 & Estonia \\
\hline \multirow[t]{2}{*}{ Poland } & & Finland & Canada & 538 & 522 & Korea \\
\hline & & $\begin{array}{l}\text { New Zealand, } \\
\text { United Kingdom, Germany, } \\
\text { Chinese Taipei, Australia, } \\
\text { Canada, Macao-China, } \\
\text { Netherlands, Switzerland, } \\
\text { Liechtenstein, Korea }\end{array}$ & $\begin{array}{l}\text { Austria, Czech Republic, } \\
\text { Belgium, Slovenia }\end{array}$ & 526 & 498 & Poland \\
\hline $\begin{array}{l}\text { Poland, Germany, Ireland, } \\
\text { Macao-China, Netherlands, } \\
\text { Liechtenstein }\end{array}$ & Korea & & & 525 & 534 & Canada \\
\hline Poland, Macao-China & & Canada & & 525 & 522 & Liechtenstein \\
\hline Poland & & $\begin{array}{l}\text { New Zealand, Chinese Taipei, } \\
\text { Canada }\end{array}$ & & 524 & 516 & Germany \\
\hline $\begin{array}{l}\text { Poland, United Kingdom, } \\
\text { Germany, Ireland, } \\
\text { Macao-China, Switzerland }\end{array}$ & & & & 523 & 532 & Chinese Taipei \\
\hline \multirow{2}{*}{$\begin{array}{l}\text { Poland, United Kingdom, } \\
\text { Ireland, Macao-China, } \\
\text { Switzerland }\end{array}$} & & Canada & & 522 & 525 & Netherlands \\
\hline & & $\begin{array}{l}\text { New Zealand, Chinese Taipei, } \\
\text { Australia, Canada, } \\
\text { Netherlands }\end{array}$ & Slovenia & 522 & 508 & Ireland \\
\hline $\begin{array}{l}\text { Poland, United Kingdom, } \\
\text { Ireland, Macao-China, } \\
\text { Switzerland }\end{array}$ & & & & 521 & 527 & Australia \\
\hline Poland & & $\begin{array}{l}\text { New Zealand, Chinese Taipei, } \\
\text { Australia, Canada, } \\
\text { Netherlands, Liechtenstein }\end{array}$ & Slovenia & 521 & 511 & Macao-China \\
\hline $\begin{array}{l}\text { Poland, United Kingdom, } \\
\text { Germany, Czech Republic, } \\
\text { Ireland, Macao-China, } \\
\text { Switzerland, Slovenia }\end{array}$ & & & & 516 & 530 & New Zealand \\
\hline \multirow[t]{2}{*}{ Poland } & & $\begin{array}{l}\text { New Zealand, Chinese Taipei, } \\
\text { Australia, Netherlands }\end{array}$ & & 515 & 512 & Switzerland \\
\hline & Poland, Ireland, Macao-China & New Zealand & & 514 & 519 & Slovenia \\
\hline Poland, Latvia & & $\begin{array}{l}\text { New Zealand, Chinese Taipei, } \\
\text { Australia, Netherlands }\end{array}$ & & 514 & 515 & United Kingdom \\
\hline $\begin{array}{l}\text { United States, Latvia, France, } \\
\text { Denmark }\end{array}$ & Poland & New Zealand & & 508 & 513 & Czech Republic \\
\hline $\begin{array}{l}\text { United States, Latvia, } \\
\text { Lithuania, France, Denmark, } \\
\text { Norway }\end{array}$ & Poland & & & 506 & 511 & Austria \\
\hline $\begin{array}{l}\text { United States, Latvia, France, } \\
\text { Denmark }\end{array}$ & Poland & & & 505 & 510 & Belgium \\
\hline Italy & & $\begin{array}{l}\text { United Kingdom, Austria, } \\
\text { Czech Republic, Hungary, } \\
\text { Belgium }\end{array}$ & Sweden & 502 & 490 & Latvia \\
\hline Portugal, Italy & & $\begin{array}{l}\text { Austria, Czech Republic, } \\
\text { Belgium }\end{array}$ & & 499 & 495 & France \\
\hline Luxembourg, Portugal, Italy & & $\begin{array}{l}\text { Austria, Czech Republic, } \\
\text { Belgium }\end{array}$ & & 498 & 496 & Denmark \\
\hline Portugal, Italy & & $\begin{array}{l}\text { Austria, Czech Republic, } \\
\text { Sweden, Hungary, Belgium }\end{array}$ & & 497 & 489 & United States \\
\hline
\end{tabular}

Note: Only countries and economies that participated in the PISA 2006 and PISA 2012 assessments are shown.

Countries and economies are ranked in descending order of their mean science performance in PISA 2012.

Source: OECD, PISA 2012 Database, Table I.5.3b

StatLink 凋s http://dx.doi.org/10.1787/888932935629 
- Figure I.5.5 [Part 3/4]

Multiple comparisons of science performance between 2006 and 2012

\begin{tabular}{|c|c|c|c|c|c|}
\hline & $\begin{array}{c}\text { Science } \\
\text { performance } \\
\text { in } 2006\end{array}$ & $\begin{array}{c}\text { Science } \\
\text { performance } \\
\text { in } 2012\end{array}$ & $\begin{array}{c}\text { Countries/economies with similar } \\
\text { performance in } 2006 \\
\text { but lower performance in } 2012\end{array}$ & $\begin{array}{c}\text { Countries/economies with similar } \\
\text { performance in } 2006 \\
\text { and similar performance in } 2012\end{array}$ & $\begin{array}{c}\text { Countries/economies with similar } \\
\text { performance in } 2006 \\
\text { but higher performance in } 2012\end{array}$ \\
\hline Spain & 488 & 496 & $\begin{array}{l}\text { Slovak Republic, Iceland, } \\
\text { Russian Federation }\end{array}$ & $\begin{array}{l}\text { United States, Croatia, Latvia, } \\
\text { Luxembourg, Lithuania, France, } \\
\text { Denmark, Norway }\end{array}$ & Poland \\
\hline Lithuania & 488 & 496 & Slovak Republic, Iceland & $\begin{array}{l}\text { United States, Croatia, Latvia, } \\
\text { Luxembourg, France, Spain, Denmark, } \\
\text { Norway, Russian Federation }\end{array}$ & Poland \\
\hline Norway & 487 & 495 & Slovak Republic, Iceland & $\begin{array}{l}\text { United States, Croatia, Latvia, } \\
\text { Luxembourg, Lithuania, France, Spain, } \\
\text { Denmark, Russian Federation }\end{array}$ & \\
\hline Hungary & 504 & 494 & & France, Sweden, Denmark & $\begin{array}{l}\text { Poland, Germany, Austria, } \\
\text { Czech Republic, Ireland, Macao-China, } \\
\text { Belgium, Switzerland }\end{array}$ \\
\hline Italy & 475 & 494 & Greece & Portugal, Russian Federation & \\
\hline Croatia & 493 & 491 & Slovak Republic, Iceland & $\begin{array}{l}\text { United States, Latvia, Lithuania, France, } \\
\text { Spain, Denmark, Norway }\end{array}$ & Poland \\
\hline Luxembourg & 486 & 491 & Slovak Republic, Iceland & $\begin{array}{l}\text { United States, Lithuania, Spain, Norway, } \\
\text { Russian Federation }\end{array}$ & Latvia \\
\hline Portugal & 474 & 489 & Greece & Russian Federation, Italy & \\
\hline Russian Federation & 479 & 486 & Greece, Slovak Republic & $\begin{array}{l}\text { United States, Luxembourg, Lithuania, } \\
\text { Portugal, Norway, Italy }\end{array}$ & Latvia, Spain \\
\hline Sweden & 503 & 485 & & Hungary & $\begin{array}{l}\text { Poland, Austria, Czech Republic, France } \\
\text { Ireland, Belgium, Denmark, Switzerland }\end{array}$ \\
\hline Iceland & 491 & 478 & & Slovak Republic & $\begin{array}{l}\text { United States, Poland, Croatia, Latvia, } \\
\text { Luxembourg, Lithuania, France, Spain, } \\
\text { Denmark, Norway }\end{array}$ \\
\hline Slovak Republic & 488 & 471 & & Iceland & $\begin{array}{l}\text { United States, Poland, Croatia, Latvia, } \\
\text { Luxembourg, Lithuania, France, Spain, } \\
\text { Denmark, Norway, Russian Federation }\end{array}$ \\
\hline Israel & 454 & 470 & Chile & & \\
\hline Greece & 473 & 467 & & & Portugal, Russian Federation, Italy \\
\hline Turkey & 424 & 463 & $\begin{array}{l}\text { Uruguay, Thailand, Jordan, Chile, } \\
\text { Serbia, Romania }\end{array}$ & Bulgaria & \\
\hline Bulgaria & 434 & 446 & Uruguay, Jordan & $\begin{array}{l}\text { Thailand, Turkey, Chile, Serbia, } \\
\text { Romania }\end{array}$ & \\
\hline Chile & 438 & 445 & Uruguay & Bulgaria, Serbia & Turkey, Israel \\
\hline Serbia & 436 & 445 & Uruguay & Bulgaria, Chile & Turkey \\
\hline Thailand & 421 & 444 & Uruguay, Jordan & Bulgaria, Romania & Turkey \\
\hline Romania & 418 & 439 & Uruguay, Jordan, Montenegro, Mexico & Thailand, Bulgaria & Turkey \\
\hline Uruguay & 428 & 416 & & Jordan & $\begin{array}{l}\text { Thailand, Turkey, Bulgaria, Chile, } \\
\text { Serbia, Romania }\end{array}$ \\
\hline Mexico & 410 & 415 & Indonesia, Montenegro & & Romania \\
\hline Montenegro & 412 & 410 & & & Mexico, Romania \\
\hline Jordan & 422 & 409 & & Uruguay & Thailand, Turkey, Bulgaria, Romania \\
\hline Argentina & 391 & 406 & Indonesia & Brazil, Tunisia, Colombia & \\
\hline Brazil & 390 & 405 & Indonesia & Argentina, Tunisia, Colombia & \\
\hline Colombia & 388 & 399 & Indonesia & Brazil, Argentina, Tunisia & \\
\hline Tunisia & 386 & 398 & Indonesia & Brazil, Argentina, Colombia & \\
\hline Qatar & 349 & 384 & & & \\
\hline Indonesia & 393 & 382 & & & $\begin{array}{l}\text { Brazil, Argentina, Tunisia, Colombia, } \\
\text { Mexico }\end{array}$ \\
\hline
\end{tabular}

Note: Only countries and economies that participated in the PISA 2006 and PISA 2012 assessments are shown.

Countries and economies are ranked in descending order of their mean science performance in PISA 2012.

Source: OECD, PISA 2012 Database, Table 1.5.3b.

StatLink तiाst http://dx.doi.org/10.1787/888932935629 
- Figure I.5.5 [Part 4/4]

Multiple comparisons of science performance between 2006 and 2012

\begin{tabular}{|c|c|c|c|c|c|c|}
\hline $\begin{array}{l}\text { Countries/economies with } \\
\text { lower performance in } 2006 \\
\text { but similar performance } \\
\text { in } 2012\end{array}$ & $\begin{array}{l}\text { Countries/economies with } \\
\text { lower performance in } 2006 \\
\text { but higher performance } \\
\text { in } 2012\end{array}$ & $\begin{array}{c}\text { Countries/economies with } \\
\text { higher performance in } 2006 \\
\text { but with similar performance } \\
\text { in } 2012\end{array}$ & $\begin{array}{l}\text { Countries/economies with } \\
\text { higher performance in } 2006 \\
\text { but lower performance } \\
\text { in } 2012\end{array}$ & $\begin{array}{c}\text { Science } \\
\text { performance } \\
\text { in } 2012\end{array}$ & $\begin{array}{c}\text { Science } \\
\text { performance } \\
\text { in } 2006\end{array}$ & \\
\hline Portugal, Italy & & Hungary & Sweden & 496 & 488 & Spain \\
\hline Portugal, Italy & & Austria, Hungary & Sweden & 496 & 488 & Lithuania \\
\hline Portugal, Italy & & Austria, Sweden, Hungary & & 495 & 487 & Norway \\
\hline \multirow{2}{*}{$\begin{array}{l}\text { United States, Croatia, Latvia, } \\
\text { Luxembourg, Lithuania, } \\
\text { Spain, Portugal, Norway, } \\
\text { Russian Federation, Italy }\end{array}$} & & & & 494 & 504 & Hungary \\
\hline & & $\begin{array}{l}\text { United States, Croatia, Latvia, } \\
\text { Luxembourg, Lithuania, } \\
\text { France, Sweden, Hungary, } \\
\text { Spain, Denmark, Norway }\end{array}$ & Slovak Republic, Iceland & 494 & 475 & Italy \\
\hline $\begin{array}{l}\text { Luxembourg, Portugal, } \\
\text { Russian Federation, Italy }\end{array}$ & & Sweden, Hungary & & 491 & 493 & Croatia \\
\hline \multirow[t]{3}{*}{ Portugal, Italy } & & $\begin{array}{l}\text { Croatia, Sweden, Hungary, } \\
\text { Denmark }\end{array}$ & & 491 & 486 & Luxembourg \\
\hline & & $\begin{array}{l}\text { United States, Croatia, } \\
\text { Luxembourg, Lithuania, } \\
\text { France, Sweden, Hungary, } \\
\text { Spain, Denmark, Iceland, } \\
\text { Norway }\end{array}$ & Slovak Republic & 489 & 474 & Portugal \\
\hline & & $\begin{array}{l}\text { Croatia, Sweden, Hungary, } \\
\text { Iceland }\end{array}$ & & 486 & 479 & Russian Federation \\
\hline $\begin{array}{l}\text { United States, Croatia, } \\
\text { Luxembourg, Israel, } \\
\text { Iceland, Portugal, Norway, } \\
\text { Russian Federation, Italy }\end{array}$ & Latvia, Lithuania, Spain & & & 485 & 503 & Sweden \\
\hline $\begin{array}{l}\text { Israel, Portugal, } \\
\text { Russian Federation }\end{array}$ & Italy & Sweden & & 478 & 491 & Iceland \\
\hline Greece, Turkey, Israel & Portugal, Italy & & & 471 & 488 & Slovak Republic \\
\hline Turkey & & $\begin{array}{l}\text { Greece, Slovak Republic, } \\
\text { Sweden, Iceland }\end{array}$ & & 470 & 454 & Israel \\
\hline \multirow[t]{3}{*}{ Turkey, Israel } & & Slovak Republic & & 467 & 473 & Greece \\
\hline & & $\begin{array}{l}\text { Greece, Slovak Republic, } \\
\text { Israel }\end{array}$ & & 463 & 424 & Turkey \\
\hline & & & & 446 & 434 & Bulgaria \\
\hline Thailand, Romania & & & & 445 & 438 & Chile \\
\hline \multirow[t]{3}{*}{ Thailand, Romania } & & & & 445 & 436 & Serbia \\
\hline & & Chile, Serbia & & 444 & 421 & Thailand \\
\hline & & Chile, Serbia & & 439 & 418 & Romania \\
\hline $\begin{array}{l}\text { Argentina, Montenegro, } \\
\text { Mexico }\end{array}$ & & & & 416 & 428 & Uruguay \\
\hline Argentina & & Uruguay, Jordan & & 415 & 410 & Mexico \\
\hline Brazil, Argentina & & Uruguay, Jordan & & 410 & 412 & Montenegro \\
\hline \multirow{6}{*}{$\begin{array}{l}\text { Brazil, Argentina, } \\
\text { Montenegro, Tunisia, } \\
\text { Colombia, Mexico }\end{array}$} & & & & 409 & 422 & Jordan \\
\hline & & $\begin{array}{l}\text { Uruguay, Jordan, } \\
\text { Montenegro, Mexico }\end{array}$ & & 406 & 391 & Argentina \\
\hline & & Jordan, Montenegro & & 405 & 390 & Brazil \\
\hline & & Jordan & & 399 & 388 & Colombia \\
\hline & & Jordan & & 398 & 386 & Tunisia \\
\hline & & Indonesia & & 384 & 349 & Qatar \\
\hline Qatar & & & & 382 & 393 & Indonesia \\
\hline
\end{tabular}

Note: Only countries and economies that participated in the PISA 2006 and PISA 2012 assessments are shown.

Countries and economies are ranked in descending order of their mean science performance in PISA 2012.

Source: OECD, PISA 2012 Database, Table I.5.3b

StatLink तiा sta http://dx.doi.org/10.1787/888932935629 
Figure I.5.6 shows the relationship between each country's/economy's average science performance in 2006 and their annualised change between 2006 and 2012. ${ }^{2}$ The correlation between performance in PISA 2006 and the annualised change is -0.39 , signalling that countries and economies that had lower performance in their first PISA science assessment are more likely to be those that improve the fastest. To put it another way, 15\% of the variation in countries'/economies' annualised change in science performance can be explained by its initial performance in PISA (Table I.5.3b). Of the 19 countries and economies that saw an improvement in science performance since PISA 2006, nine had an average initial score of 470 score points, well below the OECD average.

- Figure I.5.6

\section{Relationship between annualised change in science performance and average PISA 2006 science scores}

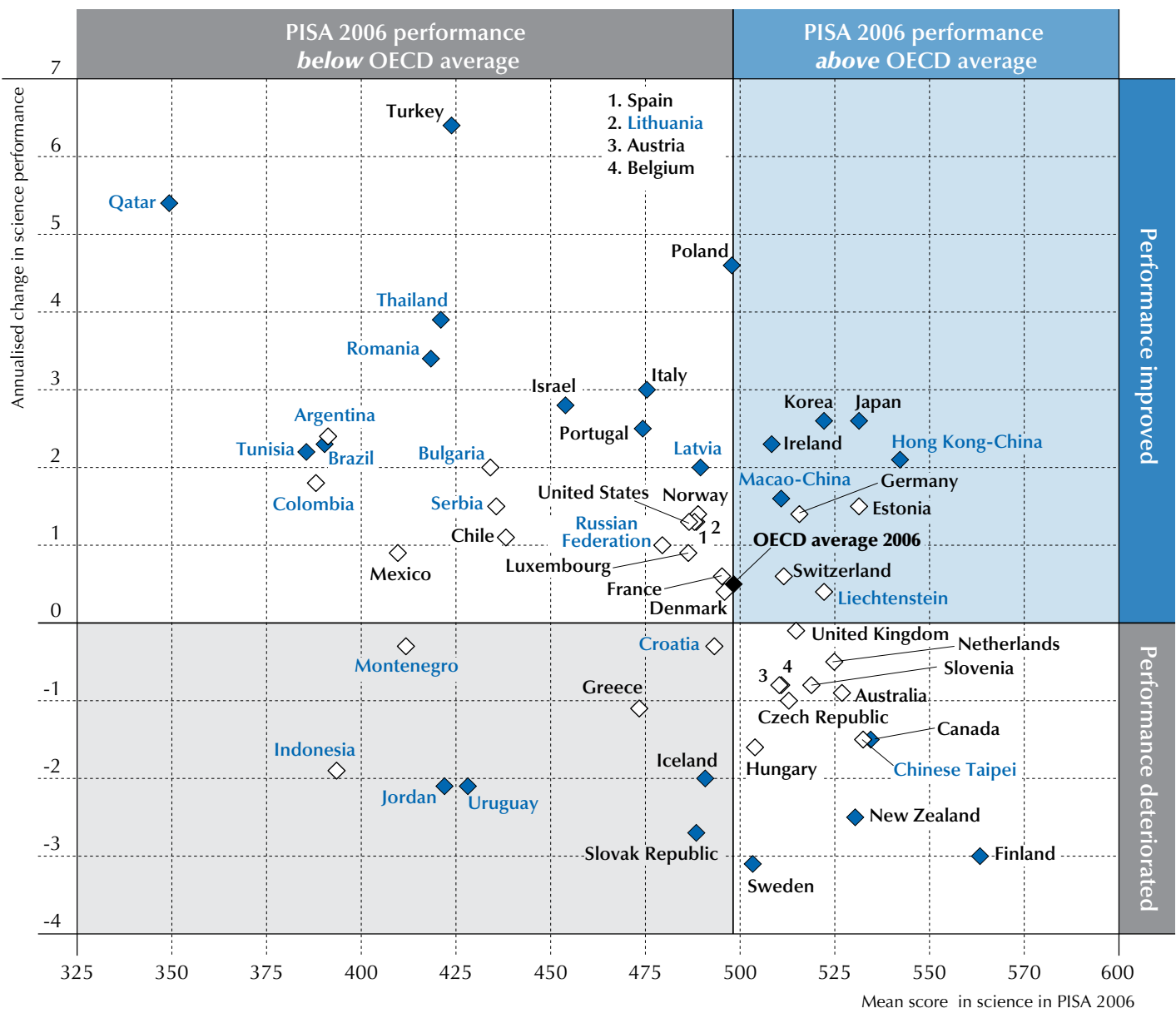

Notes: Annualised score point change in science that are statistically significant are indicated in a darker tone (see Annex A3).

The annualised change is the average annual change in PISA score points from a country/economy's earliest participation in PISA to PISA 2012. It is calculated taking into account all of a country's/economy's participation in PISA. For more details on the calculation of the annualised change, see Annex A5.

OECD average 2006 considers only those countries with comparable data since PISA 2006.

The correlation between a country's/economy's mean score in 2006 and its annualised performance is -0.39.

Source: OECD, PISA 2012 Database, Tables I.5.3b.

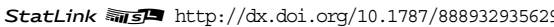

Yet it is not inevitable that only countries and economies that perform below the OECD average show improvements over time. Japan, for example, performed significantly above the OECD average in science in 2006 (at 531 points) and by 2012, shows an annualised improvement in science performance of around two score points per year. Estonia had similar levels of performance to Japan in PISA 2006 and improved, in the three years between PISA 2009 and PISA 2012 by 14 score points. Similarly, among the countries and economies that scored around the OECD average in science in 2006 , 
Poland and Ireland saw improvements by 2012 but Sweden and Hungary did not. The Russian Federation, Italy, Portugal and Greece, for example, all showed similar levels of performance in science in 2006 (around 475 points), but while Italy and Portugal improved their performance by 2012, the Russian Federation and Greece did not. Also telling is that among countries that performed below the OECD average in 2006, eight countries saw no improvement up until 2012. This underscores the fact that all countries and economies can improve their science performance, irrespective of how well they perform in science (Figure I.5.6).

\section{Trends in science performance adjusted for sampling and demographic changes}

There are many reasons why a country's or economy's science performance may change over time. Improvements may be the result of specific education policies or changes in the demographic characteristics of the population. For example, because of trends in migration, the characteristics of the PISA reference population - 15-year-olds enrolled in school may have shifted; or, as a result of economic, cultural and social development, the environments in which students live can better promote student learning. By asking students about their after-school experiences and backgrounds, PISA can identify whether the socio-economic conditions of students have changed and whether more students had an immigrant background in 2012 than did in previous years. These differences in the characteristics of the reference population may be driving the observed trends in some countries but not in others. ${ }^{3}$

Adjusted trends shed light on those trends in science performance that are not due to changes in the demographic and socio-economic characteristics of the student population. Figure I.5.7 presents the adjusted annualised change after assuming that the average age and socio-economic status of students in 2006 and 2009 is the same as that of students who took part in PISA 2012. This adjusted trend also assumes that the proportion of girls, students with an immigrant background and students who speak a language at home that is different from that of the assessment is identical in previous cycles to those observed in PISA 2012. In short, it assumes that the population and sample characteristics observed in 2012 have not changed since 2006. Countries and economies that see a difference between the adjusted trends and the observed trends, particularly when the observed trend is more negative than the adjusted trend (non-negative), can consider these changes in the student population as a challenge that needs to be addressed by the school system, as it is the observed trends, not the adjusted trends, that measure the quality and the real-life outcome of school systems.

After accounting for differences in the sampling and population characteristics, 11 countries and economies show an improvement in science performance. For these countries and economies, the annualised change in performance observed throughout their participation in PISA is not completely attributable to changes in the background characteristics of the students who take part in PISA. This means that, in these countries and economies, either the background characteristic of students haven't changed during the period, that any changes that may have taken place have not brought about differences in average performance, or that improved education services have offset any negative effect on average science performance related to changes in the population.

On average across OECD countries, for example, the observed overall annualised improvement in science performance is no longer observed after changes in students' demographic characteristics are taken into account. This means that, on average across OECD countries, improvements in science performance can be explained by changes in the background characteristics of the student population. Similarly, the annualised improvement observed in Brazil, Hong Kong-China, Ireland, Korea, Latvia, Portugal and Tunisia is no longer apparent when comparing students with similar characteristics across the different PISA assessments.

By contrast, less than $20 \%$ of the improvement observed in Dubai (United Arab Emirates), Israel, Italy, Kazakhstan and Turkey can be attributed to changes in the demographic profile of the student population. In these countries and economies, improvements in science performance remain after accounting for students' background characteristics. Although an important part of the annualised improvement observed in Japan, Poland, Qatar, Romania, Singapore and Thailand is explained by changes in the demographic characteristics of the student population, improvements are still observed when comparing students with similar characteristics in 2012 and previous PISA assessments. In these countries and economies, only part of the observed annualised trend can be attributed to changing country demographics. In Japan, for example, there was an average annual improvement in science performance of 2.6 points; but after accounting for changes in students' background characteristics, this annualised improvement remains but decreases to 2.0 science score points per year. In Macao-China, the observed annualised improvement between PISA 2006 and PISA 2012 becomes negative after accounting for demographic changes in the population. 


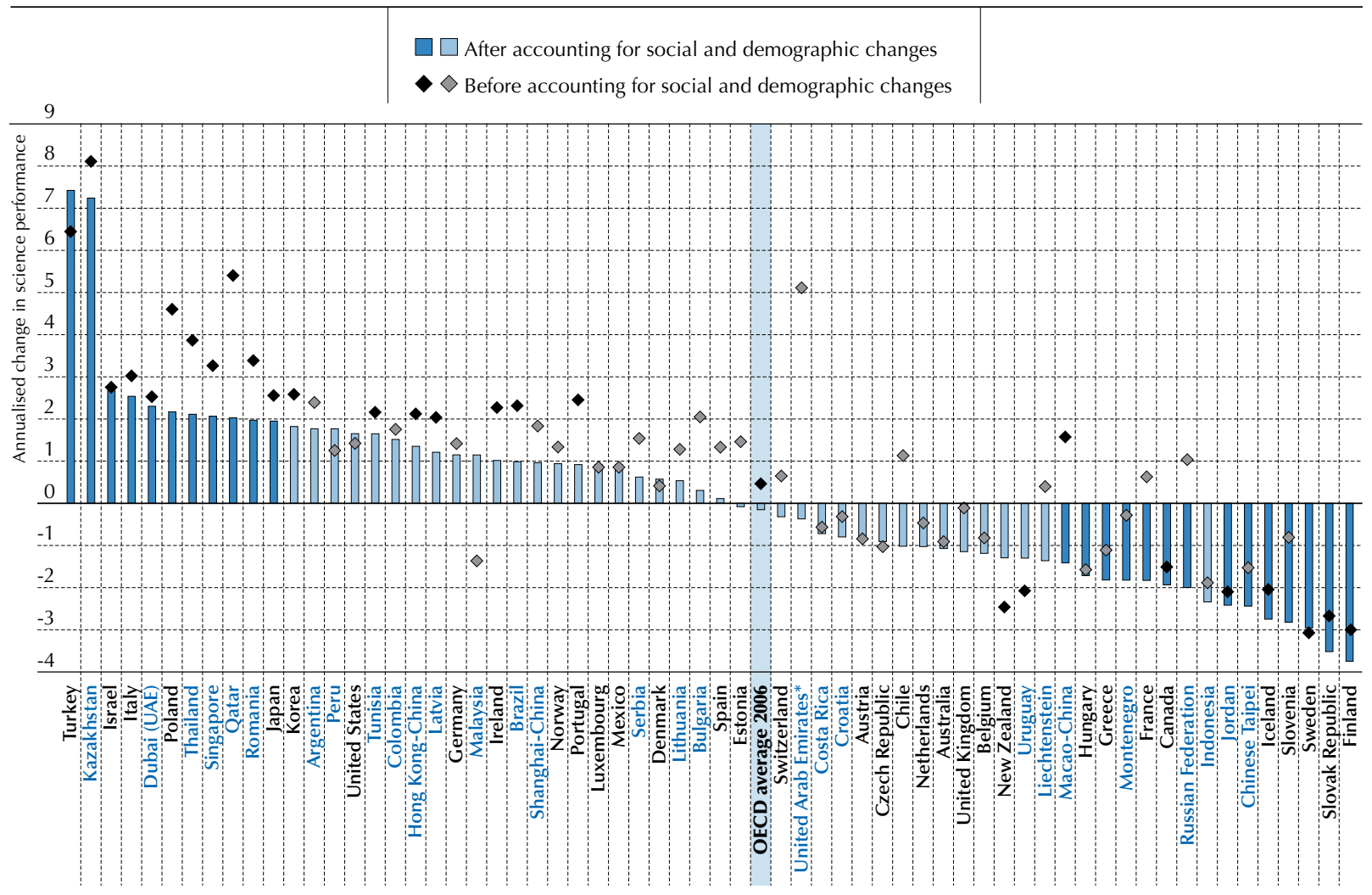

* United Arab Emirates excluding Dubai.

Notes: Statistically significant values are marked in a darker tone (see Annex A3).

The annualised change is the average annual change in PISA score points. It is calculated taking into account all of a country's/economy's participation in PISA. For more details on the calculation of the annualised change, see Annex A5.

The annualised change adjusted for demographic changes assumes that the average age and PISA index of social, cultural and economic status, as well as the percentage of female students, those with an immigrant background and those who speak a language other than the assessment at home is the same in previous assessments as those observed in 2012. For more details on the calculation of the adjusted annualised change, see Annex A5.

OECD average 2006 considers only those countries with comparable science scores since PISA 2006.

Countries and economies are ranked in descending order of the annualised change after accounting for demographic changes.

Source: OECD, PISA 2012 Database, Tables I.5.3b and I.5.4.

StatLink 形IS http://dx.doi.org/10.1787/888932935629

Informative as they may be, adjusted trends are merely hypothetical scenarios that help to understand the source of changes in students' performance over time. Observed trends depicted in Figure 1.5.7 and throughout this chapter summarise the overall evolution of a school system, highlighting the challenges that countries and economies face in improving students' and schools' science performance.

\section{Students at the different levels of proficiency in science}

When science was the major domain in PISA 2006, six proficiency levels were defined on the science scale. These same proficiency levels are used for reporting science results in PISA 2012. The process used to produce proficiency levels in science is similar to that used to produce proficiency levels in mathematics, as described in Chapter 2. Figure I.5.8 presents a description of the scientific knowledge and skills that students possess at the various proficiency levels.

Figure I.5.9 shows a map of some questions in relation to their position on the science proficiency scale. The first column shows the proficiency level within which the task is located. The second column indicates the lowest score on the task that would still be described as achieving the given proficiency level. The last column shows the name of the unit and the task number. The score given for the correct response to these questions is shown between parentheses. The selected questions have been ordered according to their difficulty, with the most difficult at the top, and the least difficult at the bottom. 


\section{- Figure I.5.8}

\section{Summary description for the six levels of proficiency in science in PISA 2012}

\begin{tabular}{|c|c|c|c|}
\hline Level & $\begin{array}{l}\text { Lower } \\
\text { score } \\
\text { limit }\end{array}$ & $\begin{array}{l}\text { Percentage of students } \\
\text { able to perform tasks } \\
\text { at each level or above } \\
\text { (OECD average) }\end{array}$ & What students can typically do \\
\hline 6 & 708 & $1.2 \%$ & $\begin{array}{l}\text { At Level 6, students can consistently identify, explain and apply scientific knowledge } \\
\text { and knowledge about science in a variety of complex life situations. They can link } \\
\text { different information sources and explanations and use evidence from those sources } \\
\text { to justify decisions. They clearly and consistently demonstrate advanced scientific } \\
\text { thinking and reasoning, and they use their scientific understanding in support of } \\
\text { solutions to unfamiliar scientific and technological situations. Students at this level } \\
\text { can use scientific knowledge and develop arguments in support of recommendations } \\
\text { and decisions that centre on personal, social or global situations. }\end{array}$ \\
\hline 5 & 633 & $8.4 \%$ & $\begin{array}{l}\text { At Level 5, students can identify the scientific components of many complex life } \\
\text { situations, apply both scientific concepts and knowledge about science to these } \\
\text { situations, and can compare, select and evaluate appropriate scientific evidence for } \\
\text { responding to life situations. Students at this level can use well-developed inquiry } \\
\text { abilities, link knowledge appropriately, and bring critical insights to situations. They } \\
\text { can construct explanations based on evidence and arguments based on their critical } \\
\text { analysis. }\end{array}$ \\
\hline 4 & 559 & $28.9 \%$ & $\begin{array}{l}\text { At Level 4, students can work effectively with situations and issues that may involve } \\
\text { explicit phenomena requiring them to make inferences about the role of science or } \\
\text { technology. They can select and integrate explanations from different disciplines of } \\
\text { science or technology and link those explanations directly to aspects of life situations. } \\
\text { Students at this level can reflect on their actions and they can communicate decisions } \\
\text { using scientific knowledge and evidence. }\end{array}$ \\
\hline 3 & 484 & $57.7 \%$ & $\begin{array}{l}\text { At Level 3, students can identify clearly described scientific issues in a range of } \\
\text { contexts. They can select facts and knowledge to explain phenomena and apply } \\
\text { simple models or inquiry strategies. Students at this level can interpret and use } \\
\text { scientific concepts from different disciplines and can apply them directly. They } \\
\text { can develop short statements using facts and make decisions based on scientific } \\
\text { knowledge. }\end{array}$ \\
\hline 2 & 409 & $82.2 \%$ & $\begin{array}{l}\text { At Level 2, students have adequate scientific knowledge to provide possible } \\
\text { explanations in familiar contexts or draw conclusions based on simple investigations. } \\
\text { They are capable of direct reasoning and making literal interpretations of the results } \\
\text { of scientific inquiry or technological problem solving. }\end{array}$ \\
\hline 1 & 335 & $95.2 \%$ & $\begin{array}{l}\text { At Level 1, students have such limited scientific knowledge that it can only be applied } \\
\text { to a few, familiar situations. They can present scientific explanations that are obvious } \\
\text { and follow explicitly from given evidence. }\end{array}$ \\
\hline
\end{tabular}

- Figure I.5.9

Map of selected science questions, by proficiency level

\begin{tabular}{|c|c|c|}
\hline Level & $\begin{array}{l}\text { Lower } \\
\text { score } \\
\text { limit }\end{array}$ & UNITS - Questions (position on PISA scale) \\
\hline 6 & 708 & GREENHOUSE - Question 5 (709) \\
\hline 5 & 633 & GREENHOUSE - Question 4.2 (659) (full credit) \\
\hline 4 & 559 & $\begin{array}{l}\text { GREENHOUSE - Question } 4.1 \text { (568) (partial credit) } \\
\text { CLOTHES - Question } 1 \text { (567) }\end{array}$ \\
\hline 3 & 484 & MARY MONTAGU - Question 4 (507) \\
\hline 2 & 409 & $\begin{array}{l}\text { MARY MONTAGU - Question } 2 \text { (436) } \\
\text { MARY MONTAGU - Question } 3(431) \\
\text { GENETICALLY MODIFIED CROPS - Question } 3 \text { (421) }\end{array}$ \\
\hline 1 & 335 & PHYSICAL EXERCISE - Question 3 (386) \\
\hline
\end{tabular}

Figure I.5.10 shows the distribution of students among these different proficiency levels in each participating country or economy. Table I.5.1 a provides figures for the percentage of students at each proficiency level on the science scale with standard errors. 
- Figure I.5.10

Proficiency in science

Percentage of students at each level of science proficiency

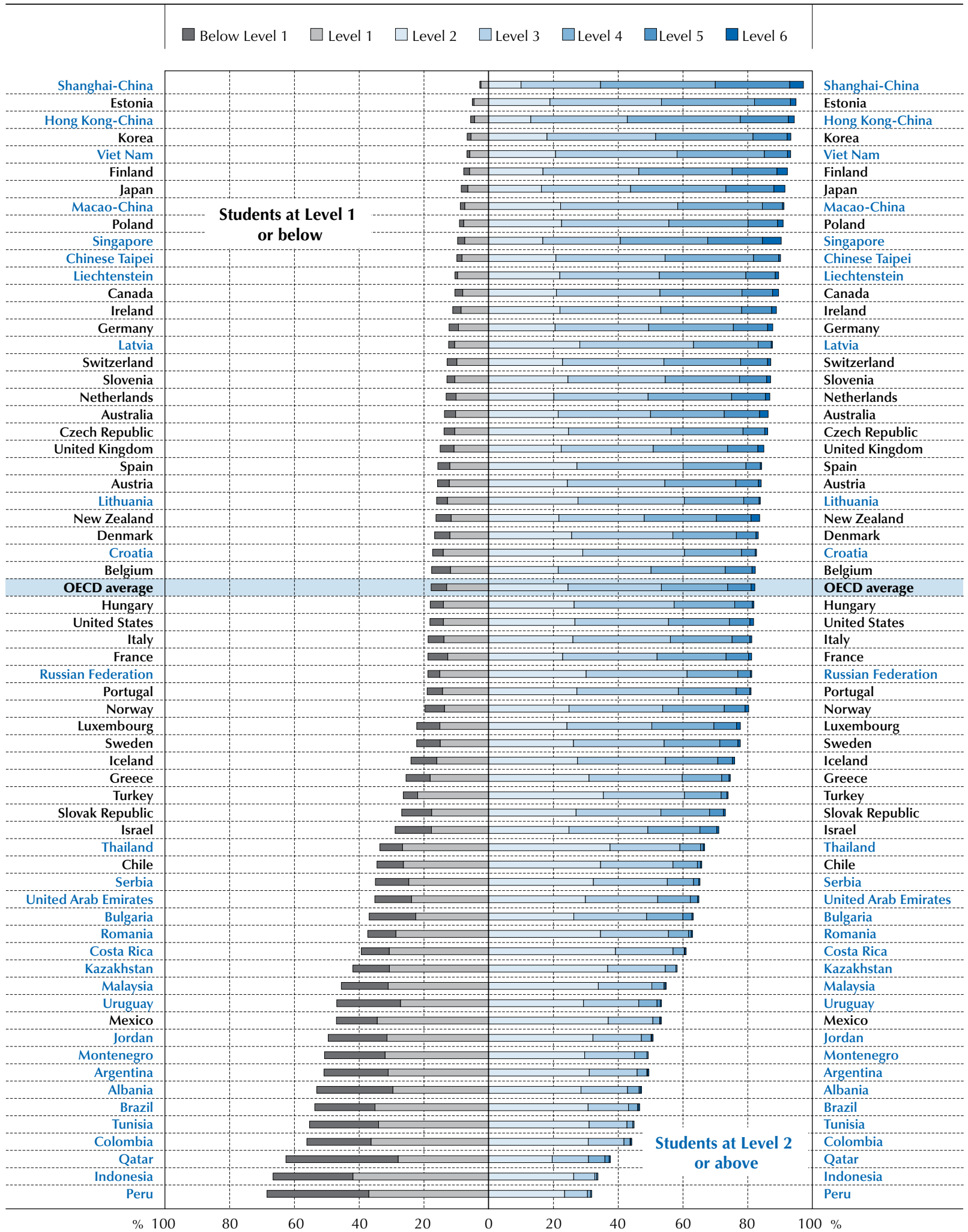

Countries and economies are ranked in descending order of the percentage of students at Levels 2, 3, 4, 5 and 6.

Source: OECD, PISA 2012 Database, Table I.5.1a.

StatLink त्राज http://dx.doi.org/10.1787/888932935629 


\section{Proficiency at Level 6 (scores higher than 708 points)}

At Level 6, students can consistently identify, explain and apply scientific knowledge and knowledge about science in a variety of complex life situations. They can link different information sources and explanations and use evidence from those sources to justify decisions. They clearly and consistently demonstrate advanced scientific thinking and reasoning, and they use their scientific understanding in support of solutions to unfamiliar scientific and technological situations. Students at this level can use scientific knowledge and develop arguments in support of recommendations and decisions that centre on personal, social or global situations.

Question 5 of GREENHOUSE (Figure 1.5.14) is an example of task at Level 6 and of the competency explaining phenomena scientifically. In this question, students must analyse a conclusion to account for other factors that could influence the greenhouse effect. This question combines aspects of the two skills: identifying scientific issues and explaining phenomena scientifically. The student needs to understand the necessity of controlling factors outside the change and measured variables and to recognise those variables. The student must have sufficient knowledge of "Earth systems" to be able to identify at least one of the factors that should be controlled. The latter criterion is considered the critical scientific skill involved, so this question is categorised as explaining phenomena scientifically. The effects of this environmental issue are global, which defines the setting.

As a first step in gaining credit for this question the student must be able to identify the change and measured variables and have sufficient understanding of methods of investigation to recognise the influence of other factors. However, the student also needs to recognise the scenario in context and identify its major components. This involves a number of abstract concepts and their relationships in determining what "other" factors might affect the relationship between the Earth's temperature and the amount of carbon dioxide emissions into the atmosphere. This locates the question near the boundary between Levels 5 and 6 in the explaining phenomena scientifically category. This question requires a short open-constructed response.

Across OECD countries, an average of $1.2 \%$ of students perform at Level 6 . Between $3 \%$ and $6 \%$ of the students are at this level in Singapore (5.8\%), Shanghai-China (4.2\%), Japan (3.4\%) and Finland (3.2\%). In New Zealand, Australia, Canada, the United Kingdom, Hong Kong-China, Estonia, Poland, Germany and Ireland between $1.5 \%$ and $2.7 \%$ of students perform at the highest proficiency level. By contrast, in the majority of participating countries the share of students at proficiency Level 6 is below 1\%. Around zero percent of students on average reach this level in Albania, Argentina, Brazil, Chile, Colombia, Costa Rica, Indonesia, Jordan, Kazakhstan, Malaysia, Mexico, Montenegro, Peru, Romania, Tunisia, Turkey and Uruguay (Figure I.5.10 and Table I.5.1a).

\section{Proficiency at Level 5 (scores higher than $\mathbf{6 3 3}$ but lower than or equal to $\mathbf{7 0 8}$ points)}

At Level 5, students can identify the scientific components of many complex life situations, apply both scientific concepts and knowledge about science to these situations, and can compare, select and evaluate appropriate scientific evidence for responding to life situations. Students at this level can use well-developed inquiry abilities, link knowledge appropriately, and bring critical insights to situations. They can construct explanations based on evidence and arguments based on their critical analysis.

Question 4 of GREENHOUSE (Figure I.5.14), an example of task at Level 5, requires an open-constructed response. This task centres on the skill using scientific evidence and asks students to identify a portion of a graph that does not provide evidence supporting a conclusion. This question requires the student to look for specific differences that vary from positively correlated general trends in these two graphical datasets. Students must locate a portion where both curves are not ascending or descending and provide this finding as part of a justification for a conclusion. As a result, the task involves a greater amount of insight and analytical skill than is required for Question 3. Rather than provide a generalisation about the relation between the graphs, the student is asked to explain the difference in the nominated period in order to gain full credit.

The question is located at Level 5 because it requires the ability to compare the details of two datasets and to criticise a given conclusion. If the student understands what the question requires of them and correctly identifies a difference in the two graphs, but is unable to explain this difference, the student gains partial credit for the question and is identified at Level 4 of the scientific proficiency scale. The skill required is to interpret data graphically presented, so the question belongs in the scientific explanations category.

Across OECD countries, $8.4 \%$ of students are proficient at Level 5 or 6 . Students scoring at Level 5 or 6 are considered as top performers. More than $15 \%$ of students attain one of these levels in Shanghai-China (27.2\%), Singapore (22.7\%), 
Japan (18.2\%), Finland (17.1\%) and Hong Kong-China (16.7\%). In 11 countries and economies between 10\% and 15\% of students are top performers in science. Some countries have virtually no top performers in science: in two partner countries, Indonesia and Peru, fewer than $0.1 \%$ of students reaches Level 5 or 6 , and in Tunisia, Colombia, Mexico, Kazakhstan, Costa Rica, Argentina, Jordan, Brazil, Malaysia, Montenegro and Albania, fewer than $0.5 \%$ of students attains Level 5 or 6 (Figure I.5.10 and Table I.5.1a).

\section{Proficiency at Level 4 (scores higher than 559 but lower than or equal to 633 points)}

At Level 4, students can work effectively with situations and issues that may involve explicit phenomena requiring them to make inferences about the role of science or technology. They can select and integrate explanations from different disciplines of science or technology and link those explanations directly to aspects of life situations. Students at this level can reflect on their actions and they can communicate decisions using scientific knowledge and evidence.

Question 1 in the unit CLOTHES (Figure I.5.15), which typifies a Level 4 question, requires the student to identify the change and measured variables associated with testing a claim about clothing. It also involves an assessment of whether there are techniques to quantify the measured variable and whether other variables can be controlled. This process then needs to be accurately applied for all four claims. The issue of "intelligent" clothes is in the category frontiers of science and technology and is a community issue addressing a need for disabled children; therefore, the setting is social. The scientific skills applied involve the nature of investigation, which places the question in the scientific enquiry category. The need to identify change and measured variables, together with an appreciation of what would be involved in carrying out measurement and controlling variables, locates the question at Level 4 . Students are required to answer in a complex multiple-choice format.

Across OECD countries, an average of $29 \%$ of students is proficient at Level 4 or higher (Level 4,5 or 6 ). In seven countries and economies, at least $40 \%$ of students attain this level, including between $40 \%$ and $50 \%$ of students in Japan, Finland, Korea, Estonia and in the partner country Singapore, slightly more than $50 \%$ in Hong Kong-China, and more than $60 \%$ of students in Shanghai-China. In contrast, fewer than $5 \%$ of students reach Level 4,5 or 6 in Indonesia, Peru, Tunisia, Colombia, Mexico, Brazil, Argentina, Jordan, Kazakhstan, Costa Rica, Albania, Malaysia and Montenegro (Figure I.5.10 and Table I.5.1a).

\section{Proficiency at Level 3 (scores higher than 484 but lower than or equal to 559 points)}

At Level 3, students can identify clearly described scientific issues in a range of contexts. They can select facts and knowledge to explain phenomena and apply simple models or inquiry strategies. Students at this level can interpret and use scientific concepts from different disciplines and can apply them directly. They can develop short statements using facts and make decisions based on scientific knowledge.

An example of a question at Level 3 is Question 4 from MARY MONTAGU (Figure I.5.16). This question requires the student to identify why young children and old people are more at risk of the effects of influenza than others in the population. Directly, or by inference, the reason is attributed to the weaker immune systems among young children and old people. The issue is community control of disease, so the setting is social. A correct explanation involves applying several pieces of knowledge that are well established in the community. The question stem also provides a clue to the groups' different levels of resistance to disease. Students have to answer with an open-constructed response.

Across OECD countries, 58\% of students are proficient at Level 3 or higher (Level 3, 4, 5 or 6 ) on the science scale. In the partner economies Shanghai-China and Hong Kong-China, more than $80 \%$ of students perform at least at this level. In the OECD countries Estonia, Finland, Korea and Japan, more than three out of four 15-year-olds are proficient at Level 3 or higher, and at least two out of three students in Singapore, Viet Nam, Chinese Taipei, Macao-China, Canada, Poland, Liechtenstein, Germany, Ireland and the Netherlands perform at least at this level (Figure I.5.10 and Table I.5.1a).

\section{Proficiency at Level 2 (scores higher than $\mathbf{4 0 9}$ but lower than or equal to $\mathbf{4 8 4}$ points)}

In 2007, following a detailed analysis of the questions from the main study, the international PISA Science Expert Group, which guided the development of the science framework and questions, identified Level 2 as the baseline proficiency level. This level does not establish a threshold for scientific illiteracy. Rather, the baseline level of proficiency defines the level of achievement on the PISA scale at which students begin to demonstrate the science competencies that will enable them to participate effectively and productively in life situations related to science and technology. At Level 2, students have adequate scientific knowledge to provide possible explanations in familiar contexts or draw conclusions based on simple investigations. They are capable of direct reasoning and making literal interpretations of the results of scientific inquiry or technological problem solving. 
Question 3 from the unit GENETICALLY MODIFIED CROPS (Figure I.5.17) is typical of Level 2 tasks. It asks a simple question about varying conditions in a scientific investigation and students are required to demonstrate knowledge about the design of science experiments. To answer this question correctly in the absence of cues, the student needs to be aware that the effect of the treatment (different herbicides) on the outcome (insect numbers) could depend on environmental factors. Thus, by repeating the test in 200 locations, the chance of a specific set of environmental factors giving rise to a spurious outcome can be accounted for. Since the question focuses on the methodology of the investigation it is categorised as scientific enquiry. The application area of genetic modification places this at the frontiers of science and technology and given its restriction to one country, it can be said to have a social setting. In the absence of cues, this question has the characteristics of Level 4, i.e. the student shows an awareness of the need to account for varying environmental factors and is able to recognise an appropriate way of dealing with that issue. However, because of the cues given in three distracters, and the fact that most students will easily eliminate these as options, the question actually sits at Level 2 of the identifying scientific issues scale.

Across OECD countries, 82\% of students, on average, are proficient at Level 2 or higher In Estonia, Hong Kong-China, Korea, Viet Nam, Finland, Japan, Macao-China, Poland, Singapore and Chinese Taipei between $90 \%$ and $95 \%$ of students perform at or above this threshold. In the partner economy Shanghai-China, only 3\% of students are below this level. In every country except the three partner countries Peru, Indonesia and Qatar, at least $40 \%$ of students are at Level 2 or above (Figure I.5.10 and Table I.5.1a).

\section{Proficiency at Level 1 (scores higher than 335 but lower than or equal to 409 points) or below}

At Level 1, students have such limited scientific knowledge that it can only be applied to a few, familiar situations. They can present scientific explanations that are obvious and follow explicitly from given evidence.

Question 3 in the unit PHYSICAL EXERCISE (Figure I.5.18) is an example of task at Level 1. To gain credit for this question, the student has to correctly recall knowledge about the operation of muscles and about the formation of fat in the body, i.e. students must have knowledge of the scientific fact that more blood flows through active muscles and that fats are not formed when muscles are exercised. This enables the student to accept the first explanation of this complex multiple-choice question and reject the second explanation. The two simple factual explanations contained in the question are not related to each other. Each is accepted or rejected as an effect of the exercise of muscles. Since this is common knowledge, the question is located at the very bottom of the explaining phenomena scientifically scale.

Students who score below 335 points - that is, below Level 1 - usually do not succeed at the most basic levels of science that PISA measures. Such students are more likely to have serious difficulties in using science to benefit from further education and learning opportunities and in participating in life situations related to science and technology (OECD, 2010).

Across OECD countries, $18 \%$ of students perform at or below Level 1 - more precisely, $13 \%$ perform at Level 1 and $5 \%$ perform below Level 1. In Shanghai-China, Estonia, Hong Kong-China, Korea, Viet Nam, Finland, Japan, Macao-China, Poland, Singapore and Chinese Taipei, fewer than $10 \%$ of students perform at Level 1 or below. In all of these countries and economies, except in Singapore $(2.2 \%), 2 \%$ of students or fewer score below Level 1 . In OECD countries, the proportion of students performing below Level 1 ranges from $2 \%$ in Japan to less than $13 \%$ in Mexico. In some countries, the share of students at proficiency Level 1 or below Level 1 is substantial, notably in Peru, Indonesia, Qatar, Colombia, Tunisia, Brazil, Albania, Argentina and Montenegro where more than half of all 15-year-olds perform at proficiency Level 1 or below. In the partner countries Qatar, Peru, Indonesia, Albania and Tunisia, more than 20\% of students perform below Level 1 (Figure I.5.10 and Table I.5.1a).

\section{Trends in the percentage of low- and top-performers in science}

PISA's science assessments gauge the extent to which a country's or economy's students have acquired the knowledge and skills in science that will allow them to participate fully in a knowledge-based society. These skills range from basic notions of science (related to proficiency Level 2) to understanding of more complex scientific concepts and processes (related to proficiency Levels 5 and 6).

Changes in a country's or economy's average performance can result from improvements or deterioration at different points in the performance distribution. For example, in some countries and economies the average improvement may be observed among all students, resulting in fewer students performing below Level 2 and more students becoming 
top performers. In other contexts, the average improvement can be attributed to large improvements among lowachieving students with little or no change among high-achieving students; this may result in a smaller share of low-performing students, but no increase in the share of top performers. From a trends perspective, countries and economies succeed when they reduce the share of students who perform below proficiency Level 2 (low performers) or when they increase the share of students who perform at or above proficiency Level 5 (top performers) as they provide more opportunities for students to begin to show scientific literacy or to have the highest level competencies in science.

Countries and economies can be grouped into categories according to whether they have: simultaneously reduced the share of low performers and increased the share of top performers between any previous PISA assessment and PISA 2012; reduced the share of low performers but not increased the share of top performers between any previous PISA assessment and PISA 2012; increased the share of top performers but not reduced the share of low performers; and reduced the share of top performers or increased the share of low performers between PISA 2012 and any previous PISA assessment. The following section categorises countries and economies into these groups.

\section{Moving everyone up: Reduction in the share of low performers and increase in that of top performers}

Between PISA 2006 and PISA 2012, Poland, Qatar and Italy saw a reduction in the share of students who perform below proficiency Level 2 in science and an increase in the share of students who perform at or above proficiency Level 5. In Poland, for example, the share of students who perform below Level 2 in science dropped from 17\% in 2006 to $9 \%$ in 2012, while the share of students who perform at or above Level 5 in science increased from $7 \%$ to $11 \%$. In Italy, 25\% of students were considered low performers in 2006; by 2012, that percentage had decreased to $19 \%$. During the same period, the proportion of top performers in Italy increased from 5\% to 6\% (Figure I.5.11). As shown in Table I.5.1b, the same was observed in Singapore, Estonia and Israel between the PISA 2009 and PISA 2012 assessments.

The reduction in the share of low performers and increase in the share of top performers in these countries and economies mirrors the changes in how students at different points of the distribution have improved since 2006. Annex B4 shows, for each country and economy, the trajectories of the 10th, 25th, 75th and 90th percentiles of science performance. These are the lowest-, low-, high- and highest-achieving students. Consistent with the changes in the shares of low and top performers, it shows how overall average improvements in Poland and Italy are also seen among their low- and high-achieving students. In Poland, for example, the lowest-achieving students improved their science performance by 5.6 score points per year (from 381 points in 2006 to 415 points in 2012), and the highestachieving students also improved their performance by an average of 3.7 points per year (from 615 points in 2006 to 637 points in 2012), resulting in a decrease in the share of students performing below Level 2 and an increase in the share of students performing at Level 5. Similar improvements in science performance among low- and highachieving students are observed in Italy and Portugal.

\section{Reducing underperformance: Reduction in the share of low performers but no change in the share of top performers}

While relatively few countries and economies succeeded in increasing the share of top performers while simultaneously reducing the share of students who do not meet the baseline proficiency in science, many reduced the share of low performers between PISA 2006 and PISA 2012. Turkey, Thailand, Romania, Tunisia, Brazil, the United States, Portugal, Latvia, Korea, Ireland, Lithuania, Spain, Japan, Switzerland and Hong Kong-China saw a reduction in the share of students performing below proficiency Level 2 between 2006 and 2012, thus raising the number of students who demonstrate science literacy. Similarly, the Czech Republic, Slovenia, Dubai (United Arab Emirates) and Kazakhstan reduced the share of low performers between PISA 2009 and PISA 2012. Latvia, Portugal, the United States, Brazil, Tunisia, Romania, Thailand and Turkey, for example, reduced the share of students performing below proficiency Level 2 by more than five percentage points between 2006 and 2012 (Figure I.5.11).

Many of the countries and economies that reduced the share of low-performing students are those that show average improvements in science, and concentrate this improvement among their low-achieving students). Annex B4 shows the trajectories of low- and high-achieving students for all countries and economies, highlighting how, in Turkey, Korea, Romania, Brazil, Chile, Estonia, Switzerland, Spain, Tunisia and Lithuania, for example, while the lowest-achieving students improved their science performance by at least two score points per year between PISA 2006 and PISA 2012, the highest-achieving students saw no change in science performance. 


\section{Nurturing top performance: Increase in the share of high-performers but no change in that of low performers}

Top-performing students in science are those who perform at or above proficiency Level 5. Luxembourg and Serbia saw an increase in the share of top-performing students while the share of low-performing students remained unchanged between 2006 and 2012. Similar improvements were observed in Albania and Macao-China. Between PISA 2009 and PISA 2012. In Luxembourg, for example, the share of top performers increased from 6\% in 2006 to $8 \%$ in 2012 (Figure I.5.11 and Table I.5.1b).

\section{Increase in the share of low performers or decrease in that of high performers}

By contrast, in 13 countries and economies the percentage of students who do not meet the baseline proficiency in science in PISA increased since 2006 - or since more recent PISA cycles - or the share of students who perform at the highest levels of proficiency decreased (Figure I.5.11 and Table I.5.1b).

\section{- Figure I.5.11}

\section{Percentage of low-performing students and top performers in science in 2006 and 2012}

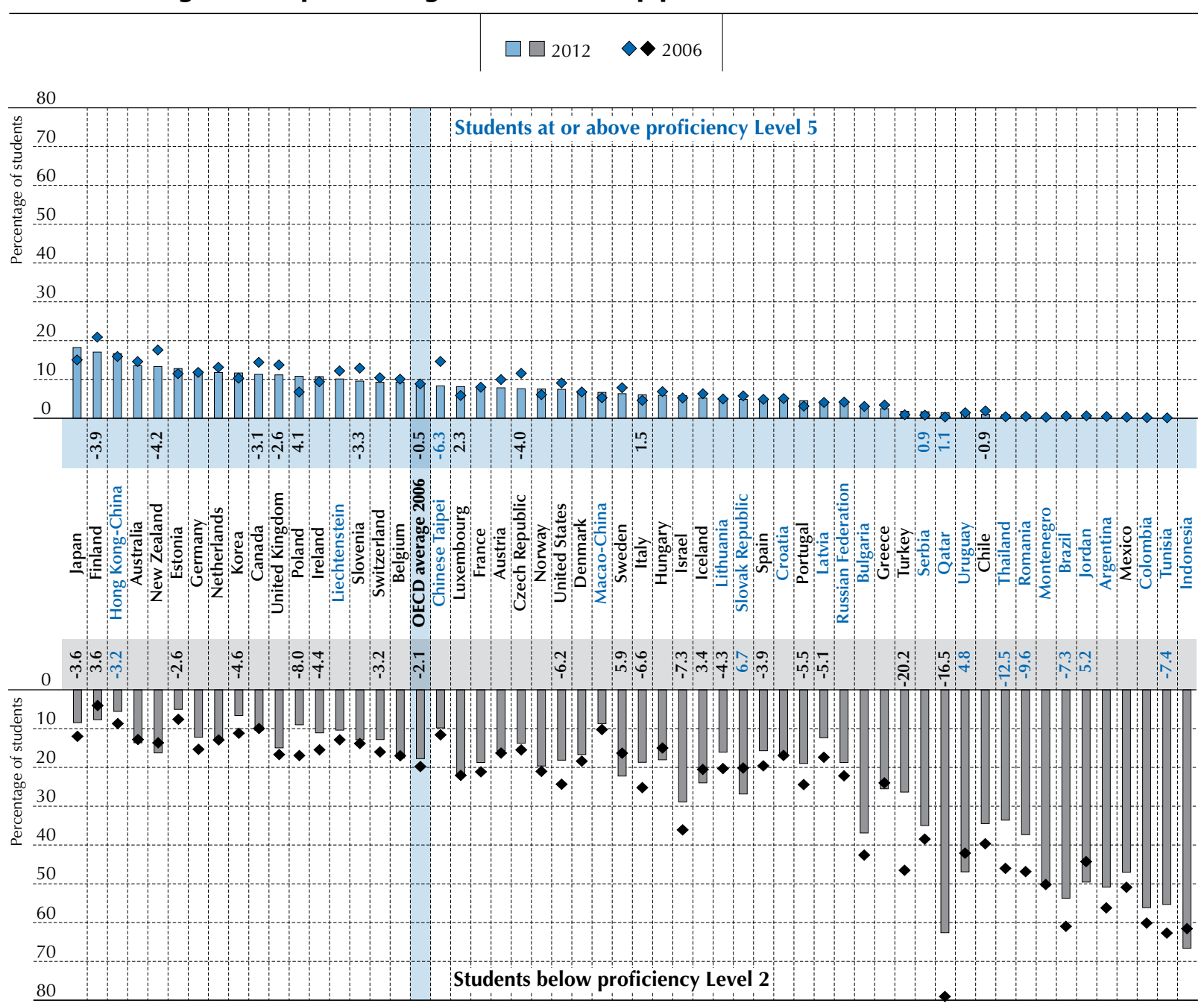

Notes: The chart shows only countries/economies that participated in both PISA 2006 and PISA 2012 assessments.

The change between PISA 2006 and PISA 2012 in the share of students performing below Level 2 in science is shown below the country/economy name. The change between PISA 2006 and PISA 2012 in the share of students performing at or above Level 5 in science is shown above the country/economy name. Only statistically significant changes are shown (see Annex A3).

OECD average 2006 compares only OECD countries with comparable science scores since 2006.

Countries and economies are ranked in descending order of the percentage of students at or above proficiency Level 5 in science in 2012

Source: OECD, PISA 2012 Database, Table I.5.1b.

StatLink त्नाजा http://dx.doi.org/10.1787/888932935629 


\section{Box I.5.1. Improving in PISA: Estonia}

Estonia's performance in PISA improved significantly since it first participated in PISA in 2006: by an average of 2.4 score points per year in reading and and science scores improved 14 points between PISA 2009 and PISA 2012. Its performance in reading improved from 501 points in PISA 2006 to 516 points in PISA 2012, and science performance improved from 531 points in PISA 2006 to 541 points in PISA 2012.

This improvement came in a challenging educational context. A significant demographic shift in Estonia's population of 1.3 million resulted in a $25 \%$ reduction in the number of students in general education between 2004 and 2012. Municipal schools in peripheral areas closed and repercussions are still being felt in teachertraining and retention systems, in higher education and in the labour market. High dropout rates further reduce the number of upper secondary and tertiary-level graduates. In addition, Estonia - as other OECD countries - faces the challenge of encouraging the best teachers to teach in remote and disadvantaged schools.

In response to the changing student population, the government changed its school funding model from a per capita to a per class criteria in 2008, allowing for a more equitable distribution of funds to rural schools, and, to reduce dropout rates, also began to promote vocational training. The change in financing recognises that not all of a schools' operational costs are variable, thus allowing many rural schools to keep functioning because in a per capita financing scheme they would have closed on budgetary reasons (Estonian Ministry of Education and Research, 2008).

To encourage newly qualified teachers to teach in small towns and rural areas, and for teachers with command of the Estonian language to teach in schools where Russian is the language of instruction, new teachers are offered an allowance of more than 12750 EUR during the first three years of teaching. Higher education institutions providing pre-service teacher training have formulated common competency standards for teachers and articulated a development plan for the teacher-training system (European Commission, 2010).

Other policy initiatives have promoted the use of assessments for self-monitoring purposes. In 2006, the Ministry of Education and Research introduced compulsory internal assessments for all pre-primary child-care institutions, general education schools and vocational training institutions, shifting supervisory functions from the state to the individual school level. Schools are offered support from the state to conduct their internal assessment (Estonian Ministry of Education and Research, 2008).

Since 2009, Estonia, through the Tiger Leap Foundation, has been promoting ICT use at all levels of education and in a wide range of study programmes, including science, mathematics, embroidery and robotics. The introduction of ICT equipment is combined with teacher training and new learning materials. For example, for mathematics projects, teachers are taught to use mathematics-learning software and funding is provided to schools to acquire computer-based algebra software (European Commission, 2010).

Based on the "Development Plan for the General Education System for 2007-2013", the national curriculum for basic and upper secondary schools was updated in January 2010 and the Basic Schools and Upper Secondary Schools Act was amended. As a result of these specifications, the volume of compulsory subjects in upper secondary schools was reduced from 72 to 63 courses and more elective courses are offered (Government of the Republic of Estonia, 2011a, 2011b).

The new national curriculum aims to offer more opportunities for a diverse student population in order to reduce grade repetition and dropout (Government of the Republic of Estonia, 2011a, 2011b). It is oriented towards learning, rather than teaching, and recognises the greater role students - and student engagement - take in the learning process. For example, in language-of-instruction classes, composition is emphasised; in natural science classes, research-based learning is promoted; in foreign-language classes, real-life situations are used to prompt responses in the language concerned. Certain topics in science and mathematics have been shifted from primary to secondary schools to ensure that they are taught in appropriate depth (Government of the Republic of Estonia, 2011a, 2011 b).

\section{Sources:}

Estonian Ministry of Education and Research (2008), The Development of Education, Estonian Ministry of Education and Research, Tallinn.

European Commission (2010), National Systems Overviews on Education Systems in Europe and Ongoing Reforms: Estonia 2010 Edition, Eurydice, Brussels.

Government of the Republic of Estonia (2011a), National Curriculum for Basic Schools, Tallinn.

Government of the Republic of Estonia (2011b), National Curriculum for Upper Secondary Schools, Tallinn. 


\section{Variation in student performance in science}

The difference in performance between students within countries and economies is shown in Table I.5.3a. Within countries, the difference in scores between the highest- (90th percentile) and lowest-achieving students (10th percentile) ranges from 174 to 281 points, with an OECD average of 239 points. Some of the lower-performing countries have among the narrowest gaps between the highest- and lowest-achieving students: Indonesia (with a gap of 174 points), Mexico (with a gap of 180 points), Colombia (with a gap of 196 points), Peru (with a gap of 200 points) and Tunisia (with a gap of 201 points). However, Viet Nam performs well above the OECD average and shows one of the ten narrowest gaps (197 points). Shanghai-China shows the best performance in science and a difference of only 209 points between the highest- and lowest-achieving students. At the other end of the spectrum, among the ten participating countries and economies that show the largest difference between the highest and lowest achievers in science, this gap ranges from between 257 to 281 points. One of the lowest-performing countries, Qatar (with a gap of 275 points), has nearly the same gap between the highest- and lowest-achieving students as one of the highest-performing countries, New Zealand (272 points). As in mathematics and reading, some countries perform well without having large differences between their highest- and lowest-achieving students. Among the eight best-performing countries in science, this is the case in Estonia, Korea, and in the partner countries and economies Viet Nam, Shanghai-China and Hong Kong-China, where the differences are around 30 points smaller than the OECD average.

\section{Gender differences in science performance}

Across OECD countries, differences in science performance related to gender tend to be small compared with the large gender gap in reading performance and the more moderate gender differences in mathematics performance. As shown in Figure 1.5.12, in more than half of the countries assessed, differences in the average score for boys and girls are not statistically significant. This indicates that gender equality is more prevalent in science performance than in mathematics or reading performance. In 2006, when science was the main focus of the PISA assessment, gender differences were observed in two of the science processes being assessed. Across OECD countries, girls scored higher in the area of identifying scientific issues, while boys outscored girls in explaining phenomena scientifically. The shorter assessment time for science in 2012 did not allow for an update of this finding.

The largest gender differences in favour of boys are observed in Colombia (18 score points) and in Luxembourg, the United Kingdom, Costa Rica, Japan and Denmark, where there is a 10-to-15 score-point difference between boys and girls. In Spain, Chile, Mexico and Switzerland, boys outperform girls in science by six to seven score points.

By contrast, in Jordan, Qatar, United Arab Emirates, girls outperform boys in science by 43, 35 and 28 score points, respectively. In Bulgaria, Thailand, Montenegro, Finland, Latvia, Lithuania, Greece, Malaysia and Turkey, girls outperform boys in science by from 20 to 10 score points (Figure I.5.12 and Table I.5.3a).

How do boys and girls differ in levels of proficiency? One way to determine this is to observe the highest level of proficiency attained by the largest group of girls and boys in each country and economy. As can be seen in Table I.5.2a, among all the participating countries and economies, the highest proficiency level attained by the largest group of boys (in 36 countries and economies) and girls (in 33 countries and economies) is Level 3 followed by Level 2 (the highest level attained by the largest group of boys in 15 countries and economies and by most girls in 21 countries and economies). But while in nine countries the highest proficiency level attained by the largest group of boys is Level 1 and in one country, below Level 1 - in six countries, Level 1 is the highest proficiency level attained by the largest group of girls. In only four countries is Level 4 the highest proficiency level attained by the largest group of boys and in five countries, the highest proficiency level attained by the largest group of girls.

On average across OECD countries, $18.6 \%$ of boys do not attain the baseline level of proficiency in science, Level 2 , and $16.9 \%$ of girls do not attain this level $-5.3 \%$ of boys and $4.2 \%$ of girls do not even attain Level 1 . The gender gap in the proportion of boys and girls performing below Level 2 is particularly pronounced in Jordan, the United Arab Emirates, Thailand, Qatar and Bulgaria. The share of girls performing below Level 2 is at least 10 percentage points smaller than that of boys. The largest difference is found in Jordan where more than $60 \%$ of boys perform at or below Level 1 compared to $39 \%$ of girls. The opposite pattern can be observed in several countries and economies. The five countries and economies with the largest gender gap, in favour of boys, among students performing below proficiency Level 2 are Colombia, Costa Rica, Liechtenstein, Luxembourg and Mexico. There appears to be no relation between overall science performance and this gender gap as these countries and economies vary considerably in overall science performance. 
- Figure I.5.12

Gender differences in science performance

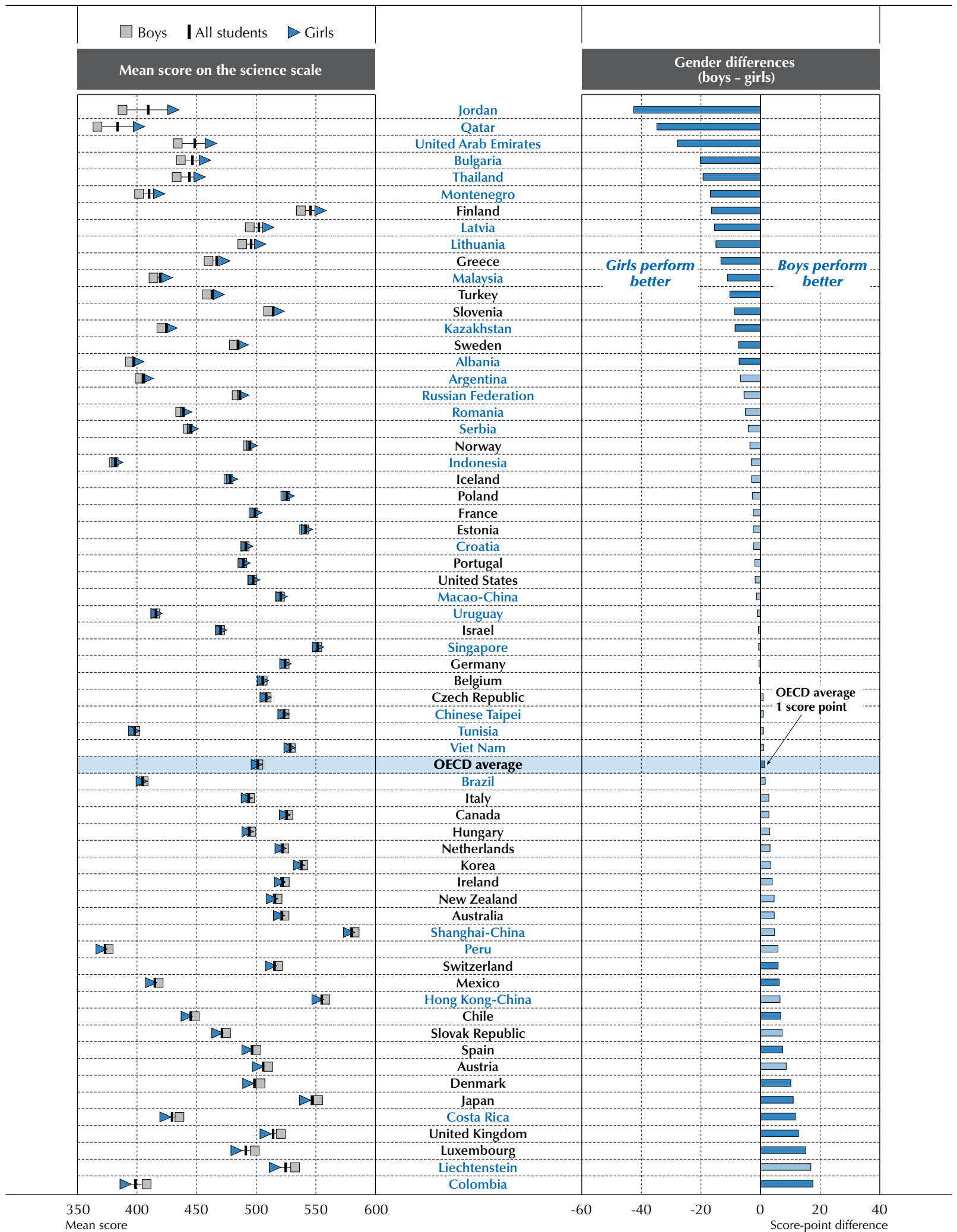

Note: Statistically significant gender differences are marked in a darker tone (see Annex A3).

Countries and economies are ranked in ascending order of the score-point difference (boys - girls).

Source: OECD, PISA 2012 Database, Table I.5.3a.

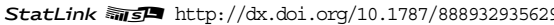


Not only do fewer girls than boys perform at the lowest proficiency levels, but fewer girls than boys perform at the highest proficiency levels on the science scale as well. Across OECD countries, $9.3 \%$ of boys are top performers in science (performing at Level 5 or 6 ), but only $7.4 \%$ of girls are.

In Japan, Liechtenstein, Hong Kong-China and Shanghai-China, all of which are among the highest-performing countries and economies in science and have relatively large shares of students performing at the highest proficiency levels, the share of top performers among boys is at least four percentage points larger than that among girls.

\section{Trends in gender differences in science performance}

In 37 of the 54 countries and economies that participated in PISA 2006 (and also took part in PISA 2012) there was no gender gap in science. A gender gap favouring boys was observed in eight countries (and largest in Chile, at 22 score points), and in ten countries, girls outperformed boys (Table I.5.3c and OECD, 2007).

Between PISA 2006 and PISA 2012, and on average across OECD countries, the gender gap in science performance remained unchanged. However, in those countries and economies where the magnitude of the gender gap in science did change, the change always favoured girls. This was the case in Finland, Montenegro, Sweden and the Russian Federation where, while there was no gender gap in science in PISA 2006, a gender gap in favour of girls was observed in PISA 2012. In the Russian Federation this is the result of an improvement in science performance among girls between PISA 2006 and PISA 2012 that was not observed among boys. In Finland, Montenegro and Sweden, the observed gender gap in science in favour of girls is the result of a greater deterioration in science performance among boys than among girls. In Chile the gender gap that favoured boys in PISA 2006 was weaker in 2012, and was no longer present in Brazil as girls' science performance has improved more rapidly than boys' (Figure I.5.13).

\section{- Figure 1.5 .13 -}

\section{Change between 2006 and 2012 in gender differences in science performance}

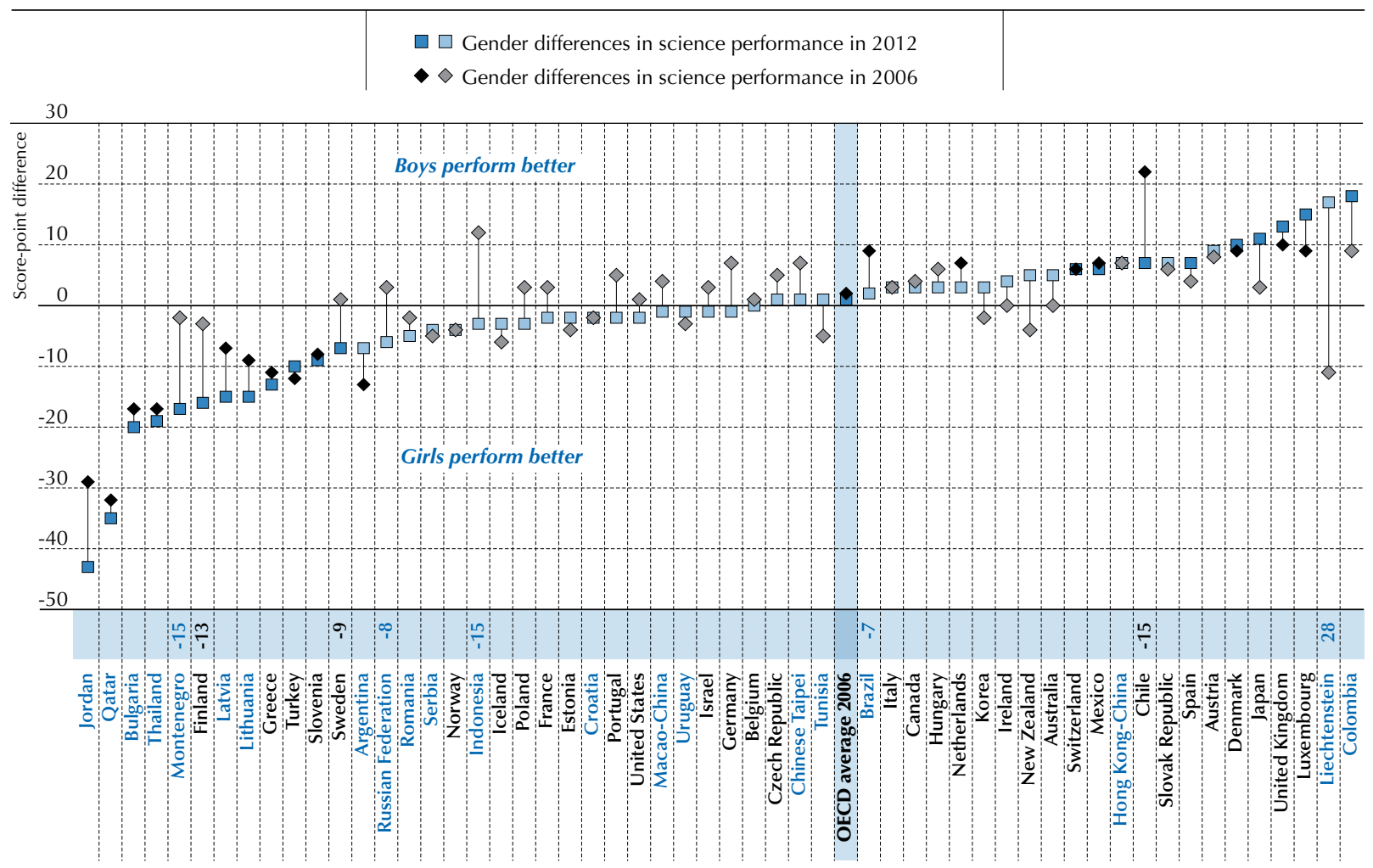

Notes: Gender differences in PISA 2006 and PISA 2012 that are statistically significant are marked in a darker tone (see Annex A3).

Statistically significant changes in the score-point difference between boys and girls in science performance between PISA 2006 and PISA 2012 are shown next to the country/economy name.

OECD average 2006 compares only OECD countries with comparable science scores since 2006. Countries and economies are ranked in ascending order of gender differences (boys-girls) in 2012.

Source: OECD, PISA 2012 Database, Table 1.5.3c.

StatLink त्राज http://dx.doi.org/10.1787/888932935629 


\section{EXAMPLES OF PISA SCIENCE UNITS}

The questions are presented in the order in which they appeared within the unit in the main survey.

\section{- Figure I.5.14}

GREENHOUSE

\section{Read the texts and answer the questions that follow.}

\section{THE GREENHOUSE EFFECT: FACT OR FICTION?}

Living things need energy to survive. The energy that sustains life on the Earth comes from the Sun, which radiates energy into space because it is so hot. A tiny proportion of this energy reaches the Earth.

The Earth's atmosphere acts like a protective blanket over the surface of our planet, preventing the variations in temperature that would exist in an airless world.

Most of the radiated energy coming from the Sun passes through the Earth's atmosphere. The Earth absorbs some of this energy, and some is reflected back from the Earth's surface. Part of this reflected energy is absorbed by the atmosphere.

As a result of this the average temperature above the Earth's surface is higher than it would be if there were no atmosphere. The Earth's atmosphere has the same effect as a greenhouse, hence the term greenhouse effect.

The greenhouse effect is said to have become more pronounced during the twentieth century.

It is a fact that the average temperature of the Earth's atmosphere has increased. In newspapers and periodicals the increased carbon dioxide emission is often stated as the main source of the temperature rise in the twentieth century.

A student named André becomes interested in the possible relationship between the average temperature of the Earth's atmosphere and the carbon dioxide emission on the Earth.

In a library he comes across the following two graphs.
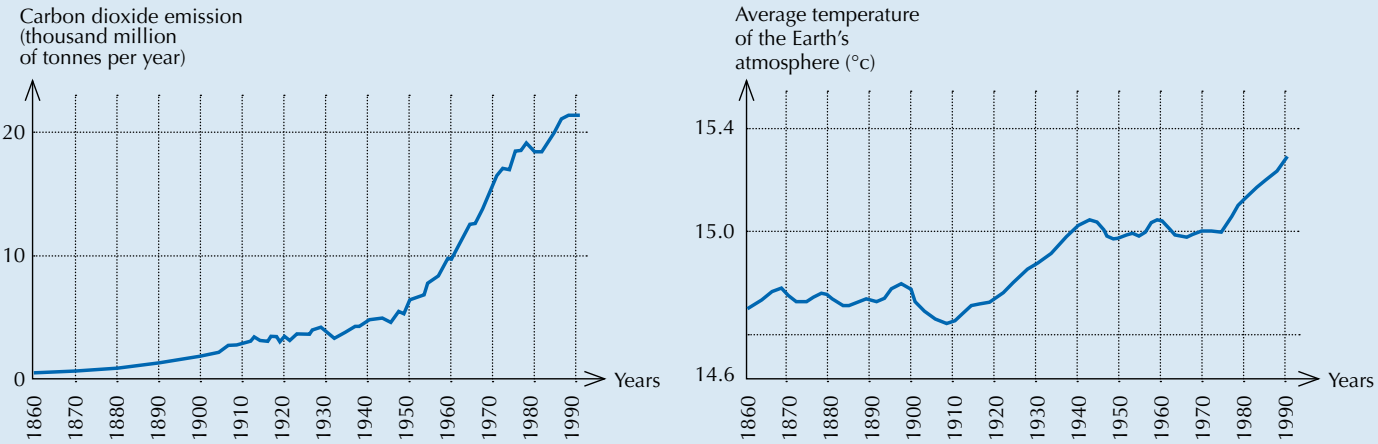

André concludes from these two graphs that it is certain that the increase in the average temperature of the Earth's atmosphere is due to the increase in the carbon dioxide emission.

\section{GREENHOUSE - QUESTION 4}

Question type: Open-constructed response

Competency: Using scientific evidence

Knowledge category: "Scientific explanations" (knowledge about science)

Application area: "Environment"

Setting: Global

Difficulty: Full credit 659; Partial credit 568 .

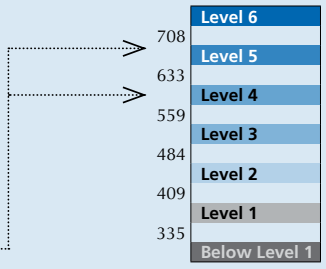

Percentage of correct answers (OECD countries): $34.5 \%$

Another student, Jeanne, disagrees with André's conclusion. She compares the two graphs and says that some parts of the graphs do not support his conclusion.

Give an example of a part of the graphs that does not support André's conclusion. Explain your answer. 
Scoring

\section{Full Credit:}

Refers to one particular part of the graphs in which the curves are not both descending or both climbing and gives the corresponding explanation. For example:

- In 1900-1910 (about) $\mathrm{CO}_{2}$ was increasing, whilst the temperature was going down.

- In 1980-1983 carbon dioxide went down and the temperature rose.

- The temperature in the 1800 s is much the same but the first graph keeps climbing.

- Between 1950 and 1980 the temperature didn't increase but the $\mathrm{CO}_{2}$ did.

- From 1940 until 1975 the temperature stays about the same but the carbon dioxide emission shows a sharp rise.

- In 1940 the temperature is a lot higher than in 1920 and they have similar carbon dioxide emissions.

\section{Partial Credit:}

Mentions a correct period, without any explanation. For example:

- 1930-1933.

- before 1910.

Mentions only one particular year (not a period of time), with an acceptable explanation. For example:

- In 1980 the emissions were down but the temperature still rose.

Gives an example that doesn't support André's conclusion but makes a mistake in mentioning the period. [Note: There should be evidence of this mistake - e.g. an area clearly illustrating a correct answer is marked on the graph and then a mistake made in transferring this information to the text.] For example:

- Between 1950 and 1960 the temperature decreased and the carbon dioxide emission increased.

Refers to differences between the two curves, without mentioning a specific period. For example:

- At some places the temperature rises even if the emission decreases.

- Earlier there was little emission but nevertheless high temperature.

- When there is a steady increase in graph 1 , there isn't an increase in graph 2 , it stays constant. [Note: It stays constant "overall".]

- Because at the start the temperature is still high where the carbon dioxide was very low.

Refers to an irregularity in one of the graphs. For example:

- It is about 1910 when the temperature had dropped and went on for a certain period of time.

- In the second graph there is a decrease in temperature of the Earth's atmosphere just before 1910.

Indicates difference in the graphs, but explanation is poor. For example:

- In the 1940s the heat was very high but the carbon dioxide very low. [Note: The explanation is very poor, but the difference that is indicated is clear.]

\section{Comment}

Another example from GREENHOUSE centres on the competency using scientific evidence and asks students to identify a portion of a graph that does not provide evidence supporting a conclusion. This question requires the student to look for specific differences that vary from positively correlated general trends in these two graphical datasets. Students must locate a portion where curves are not both ascending or descending and provide this finding as part of a justification for a conclusion. As a consequence it involves a greater amount of insight and analytical skill than is required for Question 3. Rather than a generalisation about the relation between the graphs, the student is asked to accompany the nominated period of difference with an explanation of that difference in order to gain full credit.

The ability to effectively compare the detail of two datasets and give a critique of a given conclusion locates the full credit question at Level 5 of the scientific literacy scale. If the student understands what the question requires of them and correctly identifies a difference in the two graphs, but is unable to explain this difference, the student gains partial credit for the question and is identified at Level 4 of the scientific literacy scale.

This environmental issue is global which defines the setting. The skill required by students is to interpret data graphically presented so the question belongs in the "Scientific explanations" category. 


\section{GREENHOUSE - QUESTION 5}

Question type: Open-constructed response

Competency: Explaining phenomena scientifically

Knowledge category: "Earth and space systems" (knowledge of science)

Application area: "Environment"

Setting: Global

Difficulty: 709 .

Percentage of correct answers (OECD countries): $18.9 \%$

André persists in his conclusion that the average temperature rise of the Earth's atmosphere is caused by the increase in the carbon dioxide emission. But Jeanne thinks that his conclusion is premature. She says: "Before accepting this conclusion you must be sure that other factors that could influence the greenhouse effect are constant".

Name one of the factors that Jeanne means.

\section{Scoring}

\section{Full Credit:}

Gives a factor referring to the energy/radiation coming from the Sun. For example:

- The sun heating and maybe the earth changing position.

- Energy reflected back from Earth. [Assuming that by "Earth" the student means "the ground".]

Gives a factor referring to a natural component or a potential pollutant. For example:

- Water vapour in the air.

- Clouds.

- The things such as volcanic eruptions.

- Atmospheric pollution (gas, fuel).

- The amount of exhaust gas.

- CFCs.

- The number of cars.

- Ozone (as a component of air).

\section{Comment}

Question 5 of GREENHOUSE is an example of Level 6 and of the competency explaining phenomena scientifically. In this question, students must analyse a conclusion to account for other factors that could influence the greenhouse effect. This question combines aspects of the two competencies identifying scientific issues and explaining phenomena scientifically. The student needs to understand the necessity of controlling factors outside the change and measured variables and to recognise those variables. The student must possess sufficient knowledge of "Earth systems" to be able to identify at least one of the factors that should be controlled. The latter criterion is considered the critical scientific skill involved so this question is categorised as explaining phenomena scientifically. The effects of this environmental issue are global, which defines the setting.

As a first step in gaining credit for this question the student must be able to identify the change and measured variables and have sufficient understanding of methods of investigation to recognise the influence of other factors. However, the student also needs to recognise the scenario in context and identify its major components. This involves a number of abstract concepts and their relationships in determining what "other" factors might affect the relationship between the Earth's temperature and the amount of carbon dioxide emissions into the atmosphere. This locates the question near the boundary between Level 5 and 6 in the explaining phenomena scientifically category. 


\section{- Figure I.5.15 "}

\section{CLOTHES}

Read the text and answer the questions that follow.

\section{CLOTHES TEXT}

A team of British scientists is developing "intelligent" clothes that will give disabled children the power of "speech". Children wearing waistcoats made of a unique electrotextile, linked to a speech synthesiser, will be able to make themselves understood simply by tapping on the touch-sensitive material.

The material is made up of normal cloth and an ingenious mesh of carbon-impregnated fibres that can conduct electricity. When pressure is applied to the fabric, the pattern of signals that passes through the conducting fibres is altered and a computer chip can work out where the cloth has been touched. It then can trigger whatever electronic device is attached to it, which could be no bigger than two boxes of matches.

"The smart bit is in how we weave the fabric and how we send signals through it - and we can weave it into existing fabric designs so you cannot see it's in there," says one of the scientists.

Without being damaged, the material can be washed, wrapped around objects or scrunched up. The scientist also claims it can be mass-produced cheaply.

Source: Farrer, S., "Interactive fabric promises a material gift of the garb", The Australian, 10 August 1998.

\section{CLOTHES - QUESTION 1}

Question type: Complex multiple choice

Competency: Identifying scientific issues

Knowledge category: "Scientific enquiry" (knowledge about science)

Application area: "Frontiers of science and technology"

Setting: Social

Difficulty: 567 .

Percentage of correct answers (OECD countries): $47.9 \%$

Can these claims made in the article be tested through scientific investigation in the laboratory? Circle either "Yes" or "No" for each.

\begin{tabular}{l|c}
\hline The material can be & $\begin{array}{c}\text { Can the claim be tested through scientific investigation } \\
\text { in the laboratory? }\end{array}$ \\
\hline washed without being damaged. & Yes / No \\
\hline wrapped around objects without being damaged. & Yes / No \\
\hline scrunched up without being damaged. & Yes / No \\
\hline mass-produced cheaply. & Yes / No \\
\hline
\end{tabular}

\section{Scoring}

Full Credit: Yes, Yes, Yes, No, in that order.

\section{Comment}

The question requires the student to identify the change and measured variables associated with testing a claim about the clothing. It also involves an assessment of whether there are techniques to quantify the measured variable and whether other variables can be controlled. This process then needs to be accurately applied for all four claims. The issue of "intelligent" clothes is in the category "Frontiers of science and technology" and is a community issue addressing a need for disabled children so the setting is social. The scientific skills applied are concerned with the nature of investigation which places the question in the "Scientific enquiry" category.

The need to identify change and measured variables, together with an appreciation of what would be involved in carrying out measurement and controlling variables, locates the question at Level 4. 


\section{- Figure I.5.16}

\section{MARY MONTAGU}

Read the following newspaper article and answer the questions that follow.

\section{THE HISTORY OF VACCINATION}

Mary Montagu was a beautiful woman. She survived an attack of smallpox in 1715 but she was left covered with scars. While living in Turkey in 1717, she observed a method called inoculation that was commonly used there. This treatment involved scratching a weak type of smallpox virus into the skin of healthy young people who then became sick, but in most cases only with a mild form of the disease.

Mary Montagu was so convinced of the safety of these inoculations that she allowed her son and daughter to be inoculated.

In 1796, Edward Jenner used inoculations of a related disease, cowpox, to produce antibodies against smallpox. Compared with the inoculation of smallpox, this treatment had less side effects and the treated person could not infect others. The treatment became known as vaccination.

\section{MARY MONTAGU - QUESTION 2}

Question type: Multiple choice

Competency: Explaining phenomena scientifically

Knowledge category: "Living systems" (knowledge of science)

Application area: "Health"

Setting: Social

Difficulty: 436 .

Percentage of correct answers (OECD countries): $74.9 \%$

\section{What kinds of diseases can people be vaccinated against?}

A. Inherited diseases like haemophilia.

B. Diseases that are caused by viruses, like polio.

C. Diseases from the malfunctioning of the body, like diabetes.

D. Any sort of disease that has no cure.

Scoring

Full Credit: B. Diseases that are caused by viruses, like polio.

\section{Comment}

To gain credit the student must recall a specific piece of knowledge that vaccination helps prevent diseases, the cause for which is external to normal body components. This fact is then applied in the selection of the correct explanation and the rejection of other explanations. The term "virus" appears in the stimulus text and provides a hint for students. This lowered the difficulty of the question. Recalling an appropriate, tangible scientific fact and its application in a relatively simple context locates the question at Level 2.

\section{MARY MONTAGU - OUESTION 3}

Question type: Multiple choice

Competency: Explaining phenomena scientifically

Knowledge category: "Living systems" (knowledge of science)

Application area: "Health"

Setting: Social

Difficulty: 431 .

Percentage of correct answers (OECD countries): $75.1 \%$ 
If animals or humans become sick with an infectious bacterial disease and then recover, the type of bacteria that caused the disease does not usually make them sick again.

What is the reason for this?

A. The body has killed all bacteria that may cause the same kind of disease.

B. The body has made antibodies that kill this type of bacteria before they multiply.

C. The red blood cells kill all bacteria that may cause the same kind of disease.

D. The red blood cells capture and get rid of this type of bacteria from the body.

Scoring

Full Credit: B. The body has made antibodies that kill this type of bacteria before they multiply.

\section{Comment}

To correctly answer this question the student must recall that the body produces antibodies that attack foreign bacteria, the cause of bacterial disease. Its application involves the further knowledge that these antibodies provide resistance to subsequent infections of the same bacteria. The issue is community control of disease, so the setting is social.

In selecting the appropriate explanation the student is recalling a tangible scientific fact and applying it in a relatively simple context. Consequently, the question is located at Level 2.

\section{MARY MONTAGU - QUESTION 4}

Question type: Open-constructed response

Competency: Explaining phenomena scientifically

Knowledge category: "Living systems" (knowledge of science)

Application area: "Health"

Setting: Social

Difficulty: 507 .

Percentage of correct answers (OECD countries): $61.7 \%$

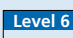

633

559 Level 4

559

Level 2

409

Level 1

Give one reason why it is recommended that young children and old people, in particular, should be vaccinated against influenza (flu).

\section{Scoring}

Full Credit: Responses referring to young and/or old people having weaker immune systems than other people, or similar. For example:

These people have less resistance to getting sick.

The young and old can't fight off disease as easily as others.

They are more likely to catch the flu.

If they get the flu the effects are worse in these people.

Because organisms of young children and older people are weaker.

Old people get sick more easily.

\section{Comment}

This question requires the student to identify why young children and old people are more at risk of the effects of influenza than others in the population. Directly, or by inference, the reason is attributed to young children and old people having weaker immune systems. The issue is community control of disease, so the setting is social.

A correct explanation involves applying several pieces of knowledge that are well established in the community. The question stem also provides a cue to the groups having different resistance to disease. This puts the question at Level 3. 


\section{- Figure I.5.17 - \\ GENETICALLY MODIFIED CROPS}

\section{GM CORN SHOULD BE BANNED}

Wildlife conservation groups are demanding that a new genetically modified (GM) corn be banned.

This GM corn is designed to be unaffected by a powerful new herbicide that kills conventional corn plants. This new herbicide will kill most of the weeds that grow in cornfields.

The conservationists say that because these weeds are feed for small animals, especially insects, the use of the new herbicide with the GM corn will be bad for the environment. Supporters of the use of the GM corn say that a scientific study has shown that this will not happen.

Here are details of the scientific study mentioned in the above article:

- Corn was planted in 200 fields across the country.

- Each field was divided into two. The genetically modified (GM) corn treated with the powerful new herbicide was grown in one half, and the conventional corn treated with a conventional herbicide was grown in the other half.

- The number of insects found in the GM corn, treated with the new herbicide, was about the same as the number of insects in the conventional corn, treated with the conventional herbicide.

\section{GENETICALLY MODIFIED CROPS - QUESTION 3}

Question type: Multiple choice

Competency: Identifying scientific issues

Knowledge category: "Scientific enquiry" (knowledge about science)

Application area: "Frontiers of science and technology"

Setting: Social

Difficulty: 421 .

Percentage of correct answers (OECD countries): $73.6 \%$

Corn was planted in 200 fields across the country. Why did the scientists use more than one site?

A. So that many farmers could try the new GM corn.

B. To see how much GM corn they could grow.

C. To cover as much land as possible with the GM crop.

D. To include various growth conditions for corn.

Scoring

Full Credit: D. To include various growth conditions for corn.

\section{Comment}

Towards the bottom of the scale, typical questions for Level 2 are exemplified by Question 3 from the unit GENETICALLY MODIFIED CROPS, which is for the competency identifying scientific issues. Question 3 asks a simple question about varying conditions in a scientific investigation and students are required to demonstrate knowledge about the design of science experiments.

To answer this question correctly in the absence of cues, the student needs to be aware that the effect of the treatment (different herbicides) on the outcome (insect numbers) could depend on environmental factors. Thus, by repeating the test in 200 locations the chance of a specific set of environmental factors giving rise to a spurious outcome can be accounted for. Since the question focuses on the methodology of the investigation it is categorised as "Scientific enquiry". The application area of genetic modification places this at the "Frontiers of science and technology" and given its restriction to one country it can be said to have a social setting.

In the absence of cues this question has the characteristics of Level 4, i.e. the student shows an awareness of the need to account for varying environmental factors and is able to recognise an appropriate way of dealing with that issue. However, the question actually performed at Level 2. This can be accounted for by the cues given in the three distractors. Students likely are able to easily eliminate these as options thus leaving the correct explanation as the answer. The effect is to reduce the difficulty of the question. 


\section{- Figure I.5.18 - \\ PHYSICAL EXERCISE}

Regular but moderate physical exercise is good for our health.

PHYSICAL EXERCISE - QUESTION 3

Question type: Complex multiple choice

Competency: Explaining phenomena scientifically

Knowledge category: "Living systems" (knowledge of science)

Application area: "Health"

Setting: Personal

Difficulty: 386 .

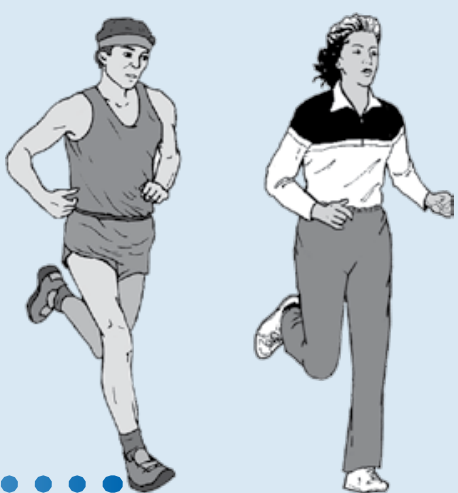

Percentage of correct answers (OECD countries): $82.4 \%$

What happens when muscles are exercised? Circle "Yes" or "No" for each statement.

\begin{tabular}{l|c}
\hline Does this happen when muscles are exercised? & Yes or No? \\
\hline Muscles get an increased flow of blood. & Yes / No \\
\hline Fats are formed in the muscles. & Yes / No \\
\hline
\end{tabular}

Scoring

Full Credit: Both correct: Yes, No, in that order.

\section{Comment}

For this question, to gain credit a student has to correctly recall knowledge about the operation of muscles and about the formation of fat in the body, i.e. students must have knowledge of the science fact that active muscles get an increased flow of blood and that fats are not formed when muscles are exercised. This enables the student to accept the first explanation of this complex multiple-choice question and reject the second explanation.

The two simple factual explanations contained in the question are not related to each other. Each is accepted or rejected as an effect of the exercise of muscles and the knowledge has widespread currency. This question is located at Level 1 , at the very bottom of the scale for the competency explaining phenomena scientifically. 


\section{Notes}

1. As described in more detail in Annex A5, the annualised change takes into account the specific year in which the assessment took place. In the case of science, this is especially relevant for the 2009 assessment as Costa Rica, Malaysia and the United Arab Emirates (excluding Dubai) implemented the assessment in 2010 as part of PISA+.

2. As described in Annex A5, the annualised change considers the case of countries and economies that implemented PISA 2009 in 2010 as part of PISA 2009+.

3. By accounting for students' gender, age, socio-economic status, migration background and language spoken at home, the adjusted trends allow for a comparison of trends in performance assuming no change in the underlying population or the effective samples' average socio-economic status, age and percentage of girls, students with an immigrant background or students that speak a language at home that is different than the language of assessment. See Annex A5 for more details on the calculation of adjusted trends.

\section{References}

Estonian Ministry of Education and Research (2008), The Development of Education, Estonian Ministry of Education and Research, Tallinn.

European Commission (2010), National Systems Overviews on Education Systems in Europe and Ongoing Reforms: Estonia 2010 Edition, Eurydice, Brussels.

Government of the Republic of Estonia (2011a), National Curriculum for Basic Schools, Tallinn.

Government of the Republic of Estonia (2011b), National Curriculum for Upper Secondary Schools, Tallinn.

OECD (2011), PISA 2009 Results: Students On Line Digital Technologies and Performance (Volume VI), PISA, OECD Publishing. http://dx.doi.org/10.1787/9789264112995-en

OECD (2010), Pathways To Success: How Knowledge And Skills At Age 15 Shape Future Lives In Canada, PISA, OECD Publishing. http://dx.doi.org/10.1787/9789264081925-en

OECD (2007), PISA 2006: Science Competencies for Tomorrow's World, PISA, OECD Publishing. http://dx.doi.org/10.1787/9789264040014-en

OECD (2006), Assessing Scientific, Reading and Mathematical Literacy: A Framework for PISA 2006, PISA, OECD Publishing. http://dx.doi.org/10.1787/9789264026407-en 


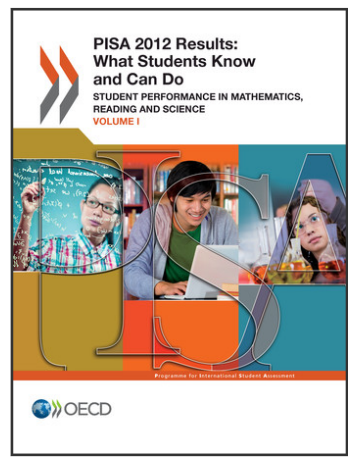

\section{From: \\ PISA 2012 Results: What Students Know and Can Do (Volume I)}

Student Performance in Mathematics, Reading and Science

Access the complete publication at:

https://doi.org/10.1787/9789264201118-en

Please cite this chapter as:

OECD (2013), "Chapter 5. A Profile of Student Performance in Science", in PISA 2012 Results: What Students Know and Can Do (Volume I): Student Performance in Mathematics, Reading and Science, OECD Publishing, Paris.

DOI: https://doi.org/10.1787/9789264201118-8-en

This work is published under the responsibility of the Secretary-General of the OECD. The opinions expressed and arguments employed herein do not necessarily reflect the official views of OECD member countries.

This document and any map included herein are without prejudice to the status of or sovereignty over any territory, to the delimitation of international frontiers and boundaries and to the name of any territory, city or area.

You can copy, download or print OECD content for your own use, and you can include excerpts from OECD publications, databases and multimedia products in your own documents, presentations, blogs, websites and teaching materials, provided that suitable acknowledgment of OECD as source and copyright owner is given. All requests for public or commercial use and translation rights should be submitted to rights@oecd.org. Requests for permission to photocopy portions of this material for public or commercial use shall be addressed directly to the Copyright Clearance Center (CCC) at info@copyright.com or the Centre français d'exploitation du droit de copie (CFC) at contact@cfcopies.com. 